\title{
Spirals and Rings in Barred Galaxies by the ROTASE Model
}

\author{
Hongjun Pan* \\ Department of Chemistry, University of North Texas, Denton, TX 76203, USA \\ *Corresponding author: hpan@unt.edu
}

\begin{abstract}
This paper extends the application of the ROTASE model for the formation of spiral arms of disc galaxies with detailed description of most common spiral patterns of barred galaxies with substantially different types of morphologies, questions and confusions from readers about this model are addressed. The optical trail effect behind the spiral arm rotation is the result of the fluid mechanism and the natural consequence of the model. The morphologies of ring galaxies are classified into four categories: type I: single ring; type II: 8-shaped double ring; type III: 8-shaped double ring wrapped by a larger outer ring; type IV: single ring without spiral and bar. All four types of ring galaxies can be described by the ROTASE model. The ROTASE model predicts that the false impression of spiral arm rotating ahead of the galactic bar in the galaxy MCG+00-04-051 will change with time, it will look like a normal galaxy with about $30^{\circ}$ to $40^{\circ}$ bar rotation in the future and the galactic bar ends will look like rotating ahead of the spiral arms with further $10^{\circ}$ to $15^{\circ} \mathrm{bar}$ rotation. The formation of one arm galaxies is due to that X-matter emission at one side of supermassive black hole is much stronger than other side. More evidence is found to support the explanation of the formation and the evolution of the Hoag's object. The possible evolution of spiral pattern of galaxies is illustrated by UGC 6093 and M51. The spiral arm winding of the Milky Way could be tighter in the future based on the ROTASE model. The possibility of spiral arm formation by the density wave mechanism and the manifold mechanism are ruled out by the one side trail effect of the galaxy J101652.52-004630.0, the special spiral pattern of the galaxy MCG+0004-051 with broken connection of spiral arm from galactic bar, the chain-link arm crossing style with sequential decrease of the luminosity along the ring lines, the special double ring pattern of the galaxy SDSS J015701.50001644.4 and the one-arm galaxy NGC 4618.
\end{abstract}

Keywords: ROTASE model, formation of spiral arms; X-matter; black hole, chain-link, ring crossing.

\section{Introduction}

If at the first the idea is not absurd, then there is no hope for it - Albert Einstein. Galaxies are the most dominant celestial massive objects in the universe. The number of galaxies in the universe may be up to 2 trillion [1], and about two thirds of those galaxies are spiral galaxies with very beautiful spiral arms and rings. Spiral galaxies have some common internal structures: a flat differential rotating disc, a stellar bugle, a super massive black hole in the center, spiral arms, a galactic bar or no bar. However, the morphologies of the spiral galaxies vary substantially among them. Hubble was the first to classify the morphologies of the spiral galaxies into different categories called Hubble sequence [2]. Elmegreen \& Elmegreen proposed a new classification scheme in which the spiral galaxies can be classified into 12 types according to the number and length of spiral arms [3,4]. It is very important to understand the mechanism of the formation of the spiral galaxies because it is related to the fate of our Milky Way and the universe. Several hypotheses have been proposed in the past. Mcvitte and Payne-Gaoschkin proposed a model based on the Newtonian gravitation laws [5]. Lin and Shu proposed the density wave theory (DWT) in 1964 [6,7], the spiral arm is treated as a quasi-stationary density wave that rotates around the galactic center with a constant pattern speed, but this theory can only be applied to a small number of the spiral galaxies with well defined architectural spiral arm patterns like the grand design galaxies. The swing amplification model describes the spiral arms as a superposition of many unstable waves, where the spirals generated by the swing amplification are transient and recurrent [8]. Romero-Gomez et. al. proposed a Manifold model in a series of 5 papers to describe the formation of the spiral and ring patterns of galaxies [9-13]. In this manifold model, the spirals and rings are developed in the vicinity of Lagragian points near the ends of galactic bars. The stars and interstellar matter circulate along the pipeline guided by the invariant manifolds. Tidal interactions may cause the formation of spiral structures also as studied by Dobbs et. al. [14]. Dobbs and Baba made detailed reviews about the spiral structure of the galaxies [15]. The author proposed a new model called Rotating Two Arm Sprinkler Emission model in 2019 (for short, ROTASE model) to describe the formation of the spiral galaxies. It was inspired by the morphological similarity between the spiral galaxies and hurricanes and between the spiral galaxies and the rotating two arm sprinkler systems used in watering lawns. A new set of spiral equations was developed and most (if not all) of spiral patterns of galaxies can be simulated. Many special characteristics were also explained by this model. The average pitch angle of the spiral galaxies can be easily calculated after the successful spiral pattern simulation [16-18]. This paper will extend the application of the ROTASE model and address questions and confusions arose since the first publication of this model. 


\section{Brief description of the ROTASE model and new spiral equations}

\subsection{Brief description of the ROTASE model}

The Rotating Two Arm Sprinkler Emission model can be briefly summarized below, and readers may refer the reference papers for the detail description of the model [16$18]$.

1. A disc galaxy can be treated as an ideal fluid system with flat differential rotation.

2. At the center of the galaxy, the central supermassive black hole (SMBH) converts the gravitational matter to unknown matter with antigravitational matter or non-gravitational matter under its extremely unimaginable physical conditions, the property of the unknown matter is unknown, and is named as X-matter for "unknown".

3. The X-matter is emitted by the SMBH in two opposite directions in the disc plane. The X-matter moves in a confined straight route for certain distance, then moves freely which will be dragged by the galactic rotation fluid, the final motion of the X-matter is the combination of the X-matter emission velocity and the galactic fluid rotation velocity.

4. The X-matter will be gradually converted to the hydrogens during its motion, such extra hydrogens increase the local hydrogen density which will promote new star formation and refuel existing local stars, temporally enhances the local luminosity which are the observed spiral arms and the galactic bar.

\subsection{The new spiral equations}

The trajectory of the X-matter can be calculated by the new spiral equations derived from the ROTASE model. The following is the primary master differential equation set:

$$
\left\{\begin{array}{c}
d x=R_{b} * \cos (\alpha) d \theta=R_{b} * \frac{y}{\sqrt{x^{2}+y^{2}}} d \theta \\
d y=R_{b} *(\rho(\theta)-\sin (\alpha)) d \theta=R_{b} *\left(\rho(\theta)-\frac{x}{\sqrt{x^{2}+y^{2}}}\right) d \theta
\end{array}\right.
$$

Where, $\mathrm{x}$ and $\mathrm{y}$ are the coordinates of spiral arm point in the calculation, precisely speaking, the $\mathrm{x}$ and $\mathrm{y}$ are the coordinates of $\mathrm{X}$-matter in the calculation. $\mathrm{R}_{\mathrm{b}}$ is the half length of the galactic bar, $\theta$ is the rotation angle of the galactic bar from the reference axis from which the $\mathrm{X}$ matter is emitted. $\alpha$ is the angle of the distance line of the $\mathrm{X}$-matter to the galactic center with respect to the reference axis from which the X-matter is emitted from the bar. The parameter $\rho(\theta)$ is defined as the ratio of the $X$-matter emission velocity $V_{e}(\theta)$ over the flat rotation velocity $V_{r}$ of the galactic fluid:

$$
\rho(\theta)=\frac{V_{e}(\theta)}{V_{r}}
$$

The rotation angle $\theta$ of the galactic bar can be used as time counting just like the clock in which the rotation angles of the clock hands can be used as time counting, the $90^{\circ}$ of the rotation angle of the minute hand represents 15 minutes of time, i.e., $90^{\circ}=15 \mathrm{~min}$. In ROTASE model, the relationship of time $t$ with the rotation angle $\theta$ is defined as:

$$
t=\frac{R_{b}}{V_{r}} \theta
$$

The $\theta$ in equation (2) represents time. The emission velocity $V_{e}$ of $X$-matter can change with time $(\theta)$ in any format, so does the $\rho(\theta)$, so the parameter $\rho$ will be the function of $\theta$ with assumption that the flat rotation velocity $\mathrm{V}_{\mathrm{r}}$ is constant. The differential equation set (1) can be solved in the polar coordinate system in three different cases with four equations: $\rho>1, \rho=1$ and $\rho<1$, respectively. Please refer the references for the detailed derivation [16-18]. For $\rho<1$, the moving distance $r$ of the $\mathrm{X}$-matter to the galactic center is limited which results in the spiral ring pattern, the radius of the ring is defined by the following equation:

$$
r(\text { radius of the ring })=\frac{R_{b}}{1-\rho}
$$

The ring radius equation (4) is very important and it is a really surprised result from this model. For $\rho=1$, the ring size is infinitive, i.e., no ring pattern; for $\rho>1$ the $r$ is unlimited. The differential equation set (1) can be called as the parent spiral equations and the four solutions can be called as the child spiral equations. The new spiral equations can be named as Galactic Spiral Equations. All angles in the Galactic Spiral Equations have the unit of radian not degree. The trajectory of the X-matter can be calculated by either the parent differential spiral equation set (1) or the child solution spiral equation set, they both give the same results, the detailed steps for the calculation are described in the references [16-18], a mini simple computer program can be used to carry such calculations. The calculated $\mathrm{x}$ and $\mathrm{y}$ have to be rotated backwardly by ($\theta$ ) for final spiral plotting with $x$ ' and $y$ ',

$$
\left\{\begin{array}{c}
x^{\prime}=x * \cos (-\theta)+y * \sin (-\theta) \\
y^{\prime}=-x * \sin (-\theta)+y * \cos (-\theta)
\end{array}\right.
$$

The critical backward rotation is clearly explained in the reference paper [18]. Euler rotation may be carried out to match the orientation of the galactic disc to the line of the sight. There are many different Euler rotation matrices available, the Euler rotation matrix used here is: 


$$
R(\varphi \sigma \psi)=\left[\begin{array}{ccc}
\cos \phi \cos \psi-\cos \sigma \sin \psi \sin \phi & -\sin \psi \cos \phi-\cos \sigma \sin \phi \cos \psi & \sin \sigma \sin \phi \\
\sin \phi \cos \psi+\cos \sigma \cos \phi \sin \psi & -\sin \phi \sin \psi+\cos \sigma \cos \phi \cos \psi & -\sin \sigma \cos \phi \\
\sin \sigma \sin \psi & \sin \sigma \cos \psi & \cos \sigma
\end{array}\right]
$$

The Galactic Spiral Equations from ROTASE model have only one variable $r$ (or $\theta$ ) and one parameter $\rho$. Therefore, they are possibly the simplest spiral formulas that can be derived from a spiral galaxy model. For now and possibly for the future, they cannot be simpler, matches Einstein's famous words: "Everything should be made as simple as possible, but not simpler", which can be called Einstein's principle of simplicity. Questions may arise: why is the proposed X-matter non-gravitational or antigravitational? Why is the X-matter emission by the SMBH in the disc plane not vertical or other angles (like the AGN emission)? Well, based on the current physics, the black hole has the super strong gravitational force, any regular gravitational matter inside of the horizon cannot escape from the black hole, therefore, the proposed X-matter must be non-gravitational or anti-gravitational. We know that all galactic bars and spiral arms are in the disc plane of the galaxies, therefore the emission of the X-matter must happen in the disc plane to fit the observations, otherwise, no such ROTASE model can be proposed. We must make the best conjecture or assumption based on all available observations and data, this is the basic philosophy adopted in all aspects of our society, not like the lottery number picking.

Such type of radiation model already exists in the universe for comparison. Figure 1 left is the illustration of the pulsar radiation, the radiation beams are emitted at the opposite directions through the south and north poles in the rotation plane which is perpendicular to the rotation axis. Figure 1 right is the illustration of the $\mathrm{X}$-matter emission by the central super massive black hole of the galaxy as proposed in this model; at this point, it is not sure if there is any physical property besides the gravitational field surrounding the outside of the SMBH which is equivalent to the magnetic field lines of the pulsar. We know little about the objects located at the centers of galaxies. The Xmatter emission is "similar" to the pulsar emission, but the scale and the mechanism totally different, we don't have any reason to exclude such emission existence in the universe. We should not limit ourselves by current knowledge. In scientific research, any conjectures and assumptions are imaginations, the imagination is the only driving force to advance the science as Einstein said: Imagination is the highest form of research. The key is to test whether the results deduced from this imagination are consistent with the broad observations. Stephen Hawking made a definition about a good theory [19]: A theory is a good theory if it satisfies two requirements. It must accurately describe a large class of observations on the basis of a model that contains only few arbitrary elements, and it must make definite predictions about the results of future observations. The conjecture made by ROTASE model will be tested through the rest of the paper with Hawking's criteria.
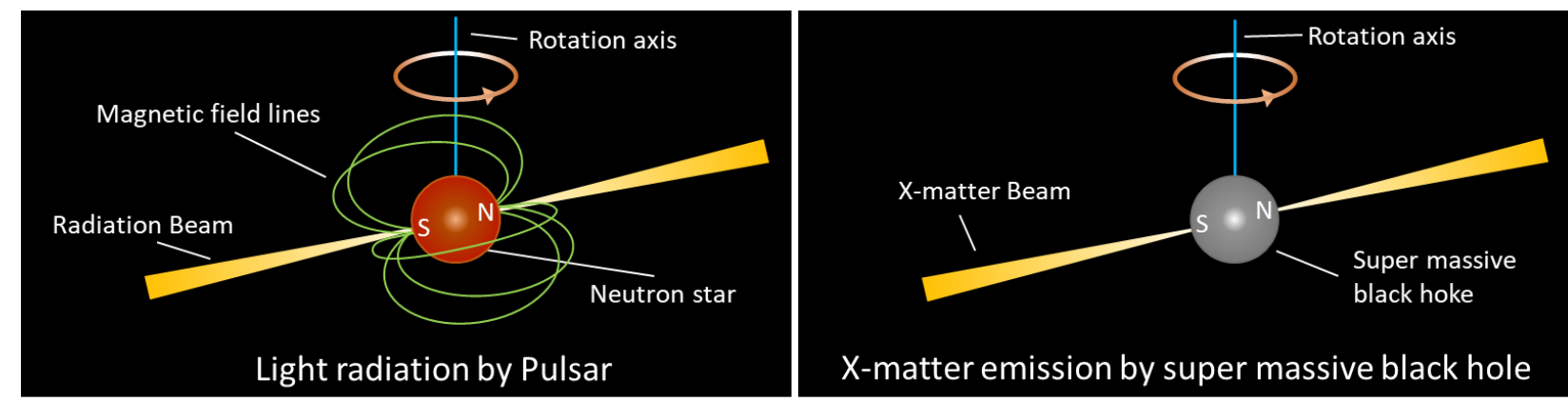

Figure 1, comparison of light radiation from a pulsar with the proposed X-matter emission from a SMBH

As the ROTASE model assumed that the amount of Xmatter will gradually decrease due to the conversion to hydrogens during its motion, less and less amount of hydrogens will be generated, this will cause the luminosity and quality of the spiral arms sequentially decrease along the spiral arm lines, which was clearly illustrated previously [17]. Such sequential decrease of luminosity and quality of the spiral arms is very important for the accurate description of the morphologies of the spiral galaxies.

The X-matter moves from inside-out, and hydrogens are created from the X-matter which will refuel the local stars and promote new star-forming during such inside-out motion. Readers may think that the stars along the spiral arm should present an age gradient from inside-out which timing should match that of the differential velocity between the propagation of the $\mathrm{X}$-matter and the disk rotation velocity. However, this is a misunderstanding. First, this misunderstanding implies that all stars in the galaxies are formed by the hydrogens converted from Xmatter. However, in the ROTASE model, the stars in the galaxies already exist before the central SMBH starts to emit the X-matter, and the hydrogens converted from the $\mathrm{X}$-matter only refuel the local stars (which already exist) and make those stars temporarily brighter, the new stars promoted by the extra hydrogens only make small portion of the total stars in the local areas. Second, the hydrogen conversion from $\mathrm{X}$-matter happens across the entire $\mathrm{X}$ matter bands simultaneously, and the star refueling and star-forming promoted by the extra hydrogens happen simultaneously in the entire X-matter band as well. This is why all new stars in the entire spiral arms have similar ages. However, the amount of hydrogens converted from the Xmatter decreases along the X-matter band lines (the spiral arm lines), so, the luminosity of the spiral arms sequentially decreases along the spiral arm lines inside-out due to the aging (decreasing the amount) of X-matter. According to the ROTASE model, the X-matter bands (the spiral arms) and the galactic bar rotate together as a "solid" object, but the galactic disc fluid rotates differentially. The newly formed stars are gravitational objects, and do not follow the 
$\mathrm{X}$-matter band (the spiral arm) rotation. They will stay with the galactic disc fluid and rotate with the fluid, will be left behind the rotation of the X-matter bands (the spiral arms), and will start their own evolution sequences. The refueled stars rotate with the galactic fluid, not with the X-matter bands. The luminosity of those refueled stars and newly formed stars will gradually decrease with gradual depletion of the extra hydrogens refueled, which will cause the observed "trail effect". As the X-matter bands continue rotation, star refueling and new star-forming continue happening in the entire X-matter bands, this completely agrees with the observations that the almost all new starforming areas are located in the spiral arm regions (except for the nuclear rings close to the galactic center) and new star-forming has a similar (narrow range of) ages in the entire arm areas. This will be further explained in the section 5 with a real galaxy.

\section{Pitch angle of spiral arms}

Pitch angle (PA) is an important parameter to describe the characteristics of the morphologies of spiral galaxies and to measure the tightness of the spiral arms. The pitch angle is the angle between the tangent of the spiral arm and the tangent of the circle of the same spiral arm location centered at the galactic center. People believe that there may be a possible relationship between the pitch angle and the mass of the central supermassive black hole; larger pitch angle may reflect smaller mass of SMBH [20-22]. From the above spiral formulas by the ROTASE model, the formula for calculating the pitch angle PA at any point ( $\mathrm{x}$ ', $\left.y^{\prime}\right)$ of the spiral arm can be derived as following:

$$
P A\left(x^{\prime}, y^{\prime}\right)=A B S\left\{\operatorname{arctg}\left[\frac{R_{b^{*}} \rho(\theta) * \cos (-\theta)+\left(1-\frac{R_{b}}{\sqrt{\left(x^{\prime}\right)^{2}+\left(y^{\prime}\right)^{2}}}\right) * x^{\prime}}{R_{b^{*}} \rho(\theta) * \sin (-\theta)-\left(1-\frac{R_{b}}{\sqrt{\left(x^{\prime}\right)^{2}+\left(y^{\prime}\right)^{2}}}\right) * y^{\prime}}\right]-\operatorname{arctg}\left[-\frac{x^{\prime}}{y^{\prime}}\right]\right\}
$$

The first arctg of the formula (7) is the tangent angle of spiral arm at location ( $\left.x^{\prime}, y^{\prime}\right)$, the second arctg of the formula (7) is the tangent angle of the circle at the same location ( $\left.x^{\prime}, y^{\prime}\right)$ with center at the galactic center. The $\mathrm{PA}\left(\mathrm{x}^{\prime}, \mathrm{y}^{\prime}\right)$ is the absolute (ABS) value of the difference of the two tangent angles, sometimes, a sign of positive or negative pitch angle is used to reflect the chirality; if the value is greater than $90^{\circ}$, then the pitch angle will be the result of subtracting the value from $180^{\circ}$. When the spiral pattern of the galaxy can be satisfactorily fit by the ROTASE model, the pitch angle will be easily calculated with equation (7). The author also first proposed a new averaging method in a previous paper [18] to more fairly average the pitch angles from all different parts of the entire galaxy, this new average method is called Spiral Arm Length Weighted Average pitch angle, for short, SALWA pitch angle PA(w). The "w" means "weighted", the PA(w) is defined as:

$$
P A(w)=\frac{1}{L} \sum P A_{i} *(d L)_{i}
$$

Where, the $P A_{i}$ is the pitch angle of $i^{\text {th }}$ data point, $(\mathrm{dL})_{i}$ is the spiral arm length at the $\mathrm{i}^{\text {th }}$ data point, $\mathrm{L}$ is the total length of the spiral arm:

$$
L=\sum(d L)_{i}
$$

The proposed SALWA pitch angle (8) is based on the fact that a different pitch angle data point $\mathrm{PA}_{\mathrm{i}}$ in the calculation represents a different length of the spiral arm section. This is true for all other commonly used spiral formulas (Archimedean, logarithmic, and Ringermacher-Mead spirals); therefore, the weighting on the length of the spiral arm section will more fairly reflect the contribution of different parts of the spiral arm for the entire galaxy. The calculation of the pitch angle based on the ROTASE model cannot quantify the error because it is based on the spiral line fitting, not based on the statistical analysis.

\section{Theoretical spiral patterns calculated by the new spiral equations}

Figure 2 lists 10 different spiral patterns calculated by the spiral equations above with different parameter $\rho$, those spiral patterns are very common in the observed galaxies. 

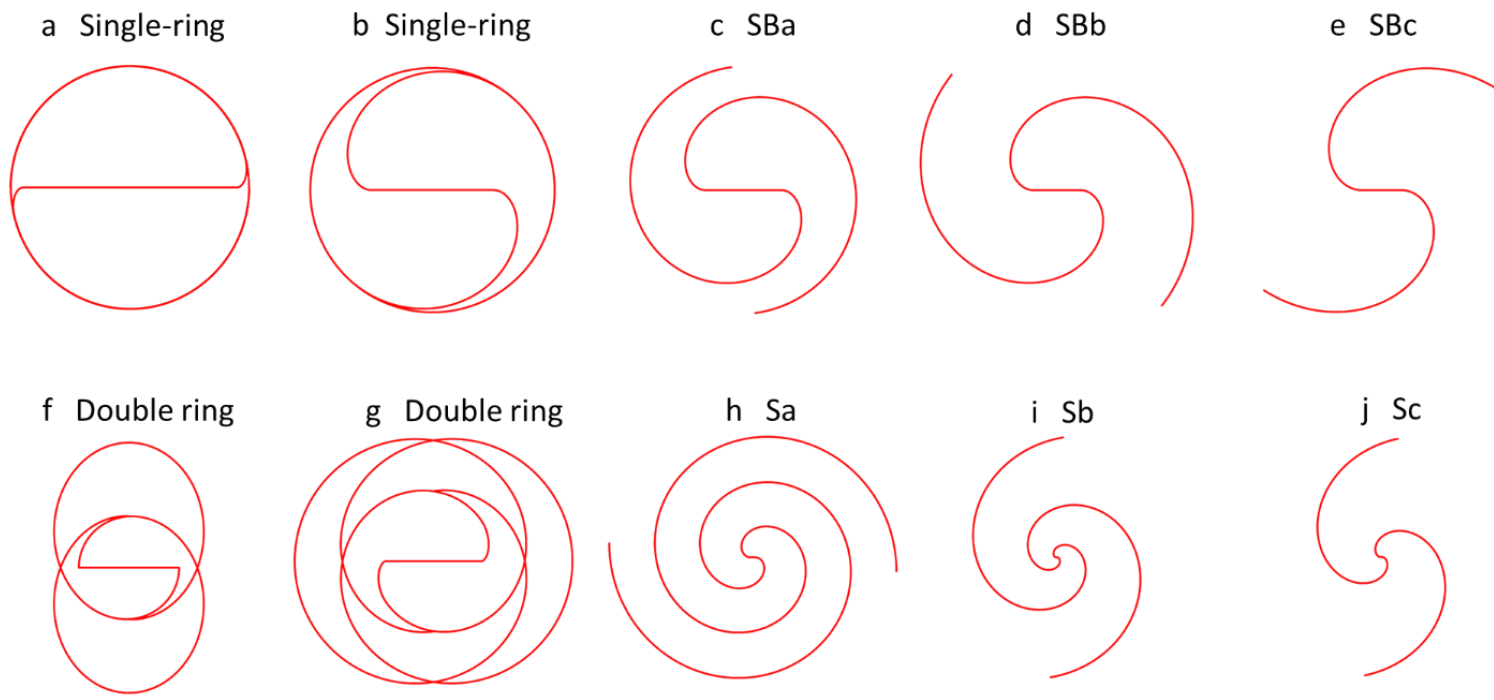

Figure 2: common galactic spiral patterns produced by the ROTASE model.

First row a: $\rho=0.1$, single spiral ring, b: $\rho=0.5$, single spiral ring; $\mathrm{c}: \rho=0.7, \mathrm{SBa} ; \mathrm{d}: \rho=1.4, \mathrm{SBb}, \mathrm{e}: \rho=2.5, \mathrm{SBc}$.

Second row f: $\rho=0.6 * \exp \left(-0.0001 *(\theta-360)^{2}\right)$, double ring; g: $\rho=0.6 * \exp \left(-0.00001 *(\theta-450)^{2}\right)$, double ring; h: $\rho=6$, Sa; i: $\rho=6+0.05 * \theta, \mathrm{Sb} ; \mathrm{j}: \rho=6+0.08 * \theta$, Sc.

Figure $2 \mathrm{a}$ is the single spiral ring pattern with constant $\rho$ $=0.1$. This is a very tight ring with little spiral structure. Figure $2 b$ is the single spiral-ring pattern with $\rho=0.5$. It has a good portion of spiral structure, and one can see that the spiral portion will increase with the parameter $\rho$. Figure $2 \mathrm{c}$ is a spiral pattern with constant $\rho=0.7$. Such a pattern is classified as SBa pattern according to Hubble sequence. It is a barred spiral with tight winding; however, with this $\rho$ value, it should be a spiral ring pattern if the spiral arm extends longer, so it is a truncated spiral ring pattern. Figure $2 \mathrm{~d}$ is the $\mathrm{SBb}$ pattern in Hubble sequence with $\rho=1.4$. It is a barred spiral with loose winding. Figure $2 \mathrm{e}$ is the $\mathrm{SBc}$ pattern in Hubble sequence with $\rho=2.5$. It is a barred spiral with more open winding. One can see that the spiral winding will be looser and more open with increase of $\rho$. Figure $2 \mathrm{f}$ is a double ring pattern in which the $\rho$ changes with time following the Gaussian equation with maximum value of $\rho$ happened at $360^{\circ}$ galactic bar rotation time ago from now. This means that peak emission of the X-matter by the central $\mathrm{SMBH}$ happened $360^{\circ}$ galactic bar rotation time ago. This type of double ring spiral pattern shows an 8 -shaped double ring structure. Figure $2 \mathrm{~g}$ is the double ring spiral pattern in which the $\rho$ changes with time following the Gaussian equation with maximum value of $\rho$ happened at $450^{\circ}$ galactic bar rotation time ago from now. This type of double ring shows an 8-shaped inner structure wrapped with a larger outer ring, and the spiral winding is much more complicated. The double ring patterns are very interesting and will be addressed later in more detail. Figure $2 \mathrm{~h}$ is the Sa pattern in Hubble sequence with $\rho=6$. It is a tight winding and unbarred spiral. Figure $2 \mathrm{i}$ is the $\mathrm{Sb}$ pattern in Hubble sequence with $\rho$ changing linearly with time. It is a loosely winding and unbarred spiral. Figure $2 \mathrm{j}$ is $\mathrm{Sc}$ pattern in Hubble sequence with $\rho$ changing with time more aggressively. The unbarred spiral galaxies may still have bars, but the length of the bars is small compared to the size of the galactic discs and cannot be recognized easily due to bright central bugle and the nuclear ring with very active new star forming.

\section{Galaxy J101652.52-004630.0 with constant X-matter emission}

The spiral galaxy J101652.52-004630.0 is a well-defined grand-design spiral galaxy with two short and very clean spiral arms shown by the original image in Figure 3 left without spurs, branches and random noisy spots to spoil the image of the galaxy. The two spiral arms have perfect central symmetry, and it should be a good standard test example for any theory of spiral arm formation of galaxies, it will be studied in detail by the ROTASE model. To the author's limited knowledge, this galaxy is relatively new to us, and has not been studied previously by other people. The spiral pattern of this galaxy was nicely simulated in a previous paper by the ROTASE model shown in Figure 3 right with solid red (north arm), orange (bar) and yellow (south arm) lines with a constant parameter $\rho=2.5$ and $95^{\circ}$ of total bar rotation angle [18]. It is a very loose open spiral galaxy and should be a SBc type galaxy in Hubble sequence. This image is almost a face-on image according to the Euler angles. This galaxy will be revisited in this paper with exploration of more details by the ROTASE model. 

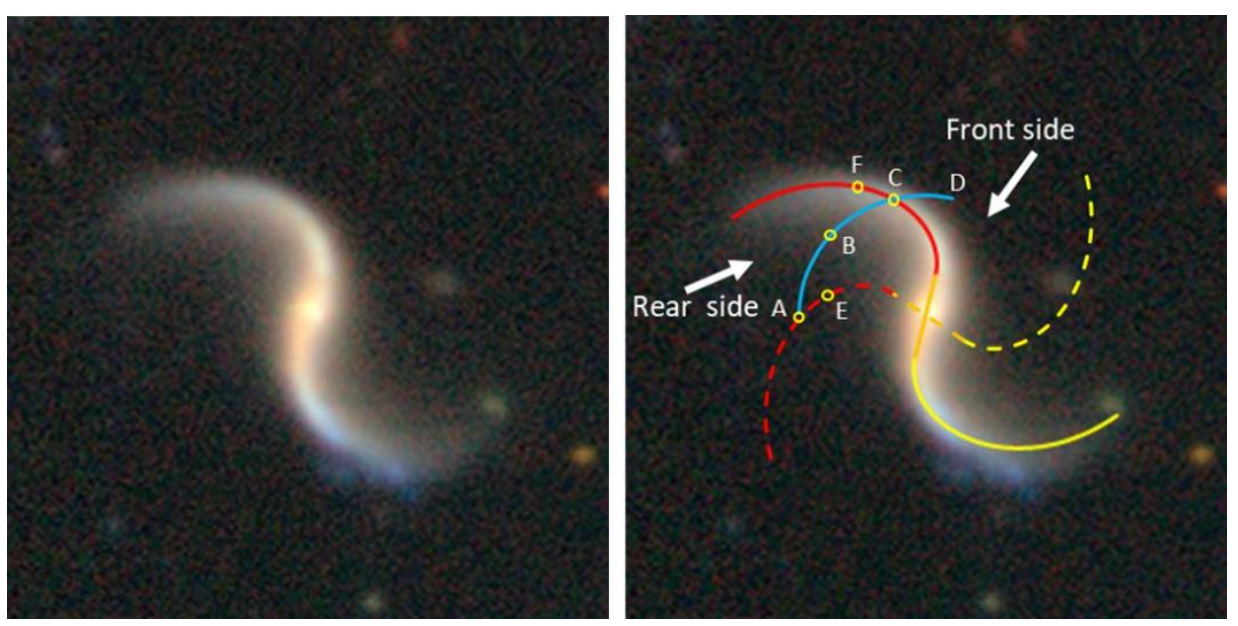

Figure 3: the galaxy J101652.52-004630.0, left: the original image, right: simulation (red and yellow) of by ROTASE model, $\rho=2.5$, Euler $(14,5,0)$

If we inspect the original image carefully, we can find: 1. The luminosity sequentially decreases along the spiral arm lines from the ends of the galactic bar to the ends of the visible arms. 2. It rotates clockwise, the front rotation side of the spiral arms has a very clean and sharp edge where the luminosity (surface brightness) decreases sharply; by contrast, the rear side is very blurry/dispersed, and the luminosity decreases slowly. The surface luminosity along the north spiral arm line (red) can be extracted as shown in Figure 4.

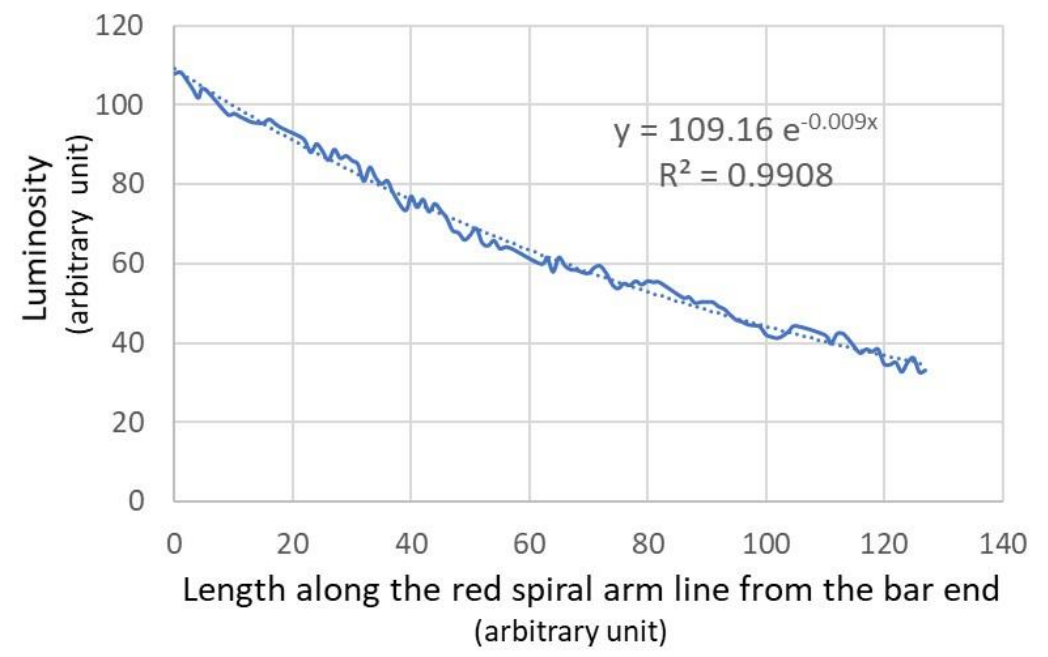

Figure 4: the surface luminosity changes along the spiral arm line in galaxy J101652.52-004630.0

One can see that the surface luminosity steadily decreases along the spiral arm line from the end of the bar to the end of the visible arm line. The decrease of the luminosity along the spiral arm line can be well fitted by an exponential decay function:

$$
y=109.16 * \exp (-0.009 * x)
$$

This equation (10) suggests that the $\mathrm{X}$-matter conversion may follow the first order conversion rate [17]. 


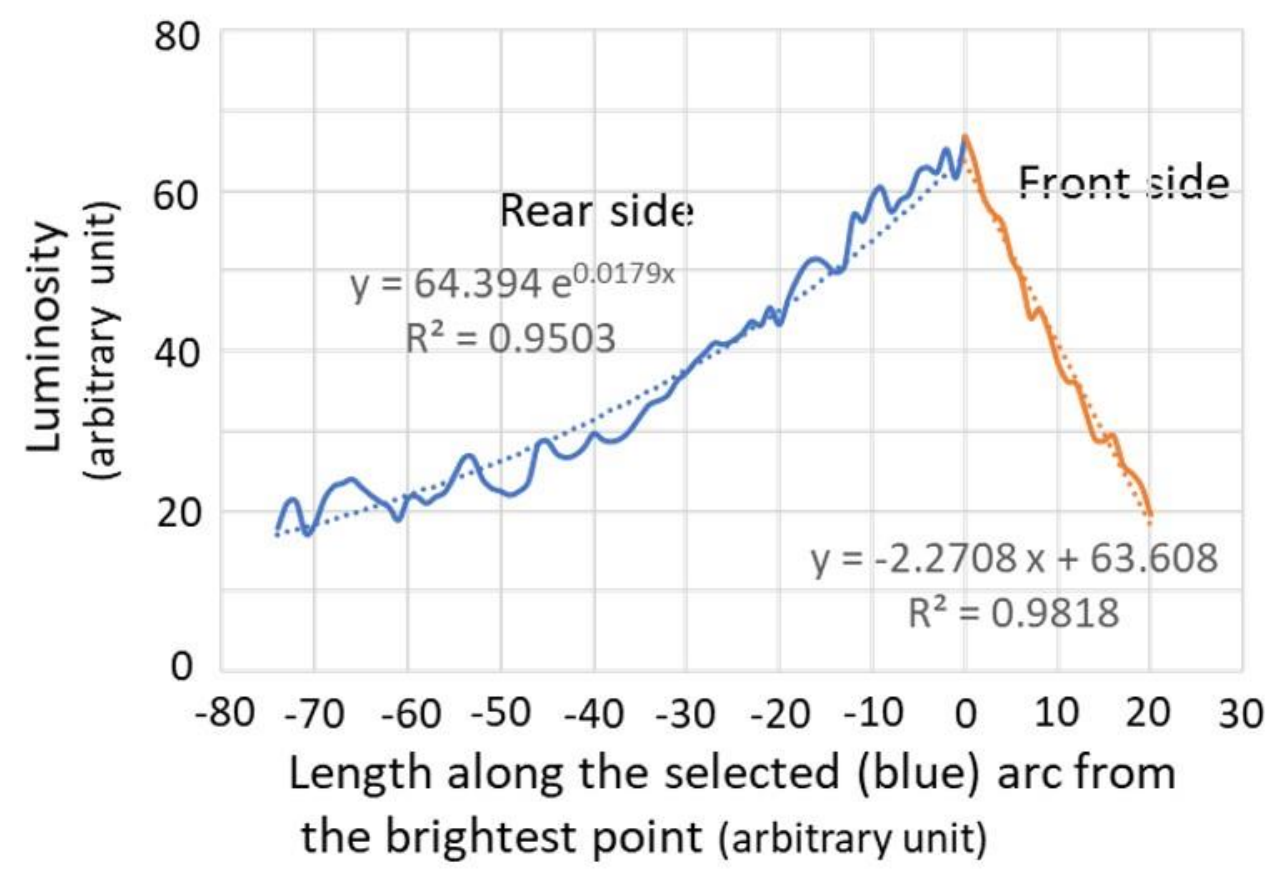

Figure 5: the surface luminosity changes along the selected blue arc in the Figure 3.

Figure 5 shows the changes of the surface luminosity along the selected blue arc (a section of a circle) from points A to D in Figure 3 right. The cross point labeled with letter $\mathrm{C}$ by the red spiral arm and the blue arc has the highest luminosity, which is defined as reference point 0 in the horizontal axis in Figure 5. For simplicity, assume that the galactic disc has a perfect differential flat rotation, and all stars and galactic matter rotate along the perfect circular lines with their radii. Based on the ROTASE model, the spiral arms and the galactic bar rotate together as a "rigid" solid structure, but the stars and other galactic matter rotate along the circular lines with their own differential rotation patterns. The spiral arm lines along the red and the yellow lines have the highest luminosity, which means that the density of X-matter is highest with the highest density of hydrogens converted. The stars in these line areas will be refueled with more hydrogens than any other areas and new star-forming will be more active than any other areas. This happens across the entirety of the X-matter band lines simultaneously. Therefore, all new stars formed in the arm lines have similar (narrow range of) ages. Due to the fact that the stars and new-born stars are gravitational, they will follow the disc fluid to rotate along the circular lines. The solid red and yellow spiral lines in Figure 3 right show the current location of spiral arms; the galaxy rotates clockwise. Therefore, at some time ago (possibly millions of years ago), spiral arms were shown in dashed red and yellow lines in Figure 3 right. The local stars in location A at that time received extra hydrogens and new stars were forming at the location A; the luminosity at location A was the highest at that time. However, as the X-matter band keeps spirally moving forward, the stars and the new-born stars at the location A also rotated along the circular (blue) line, but is behind the rotation of the X-matter band. When the X-matter band moves to its current location, the stars and new-born stars originally at the location A may have arrived at the location B with less luminosity due to gradual depletion of the extra hydrogens. Therefore, there is a luminosity gradient from the current location $\mathrm{C}$ to the location A along the circular blue line. Beyond the location
A, the luminosity is back to normal level as all extra hydrogens are depleted. This is the "trail effect" as mentioned above. The trail effect is a natural consequence of the ROTASE model, it is the result of fluid mechanism. The trail effect is very common in galaxies. The blue line (rear side) in Figure 5 is the luminosity extracted along the blue circular arc of the Figure 3 right from the cross-point $\mathrm{C}$ to A. The luminosity change along this arc can be fitted by an exponential decay function:

$$
y=64.5 * \exp (-0.018 * x)
$$

Such exponential decay dependence suggests that the depletion of the extra hydrogens may follow the first order consumption rate overall.

One has to keep in mind that as the X-matter band moves spirally outward, the X-matter originally located at location $A$ at millions of years ago has arrived at location F now, not at $\mathrm{C}$. The amount of X-matter gradually decreases along a moving line from A to F. So at F, less hydrogens will be converted, and the local stars at $\mathrm{F}$ will receive less extra hydrogens and there will be less new stars formation. The luminosity will also be weaker. The X-matter at the location $\mathrm{C}$ is not the same $\mathrm{X}$-matter at location $\mathrm{A}$, it is from "younger" X-matter at the location $\mathrm{E}$ which was closer to the galactic center in the past. The X-matter at F and $\mathrm{C}$ produce hydrogens at the same time and promote new starforming at the same time. This explains why the newly formed stars in the entire spiral arm have similar ages and should clarify the misunderstanding. The orange line in Figure 5 is the luminosity of the front side of the red spiral arm line in Figure 3 right along the blue circular arc C to D. The luminosity linearly decreases sharply compared to the rear side. Such sharp decrease reflects that the sharp front edge of the spiral arm is the sharp edge of the X-matter band with no trail effect as expected by the ROTASE model. Any successful theory for the formation of the spiral arms of galaxies must be able to clearly explain the one-side trail effect. Figure 6 shows a typical illustration from internet for the formation of spiral arms of disc galaxies by density 
wave theory. The spiral arms are formed by the special alignment of the closed elliptical orbits with $\Omega-\mathrm{n} \kappa / \mathrm{m}$, where $\Omega$ is the circular frequency and $\kappa$ is the epicyclic frequency, $n$ and $m$ are integers. Based on DWT, the spirals are not material, they are the result of density waves, the stellar matters and stars constantly enter the waves and exit

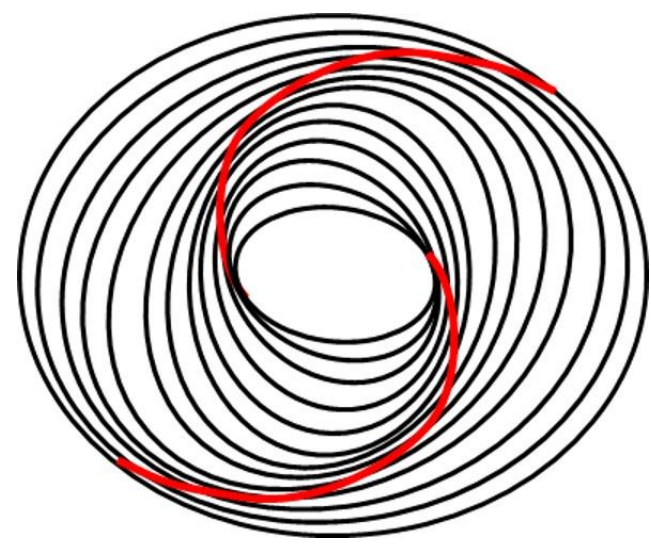

Figure 6: illustration of the spiral arm formation by density wave theory the waves. It would be expected that the trail effect of luminosity gradients should appear in both sides of the spiral arms from such in-and-out dynamic movement. However, the one side trail effect in galaxy J101652.52004630.0 rules out the possibility of spiral arm formation by the DWT and the manifold theory.

\section{Galaxy M51 with two different X- matter emissions}

The galaxy M51 is a famous whirlpool galaxy with a grand design structure shown in Figure 7, each arm has two very distinguishable spiral structures simulated in a previous paper by the yellow lines and the red lines, respectively. Overall, the two arms have almost perfect central symmetry except for the area indicated by the white arrow in which it is not fitted well. It is a good example for illustration of X-matter emission behavior change by the central SMBH and the central symmetry of the galaxy being damaged by unsymmetrical strong gravitational interaction, so it will be revisited here.

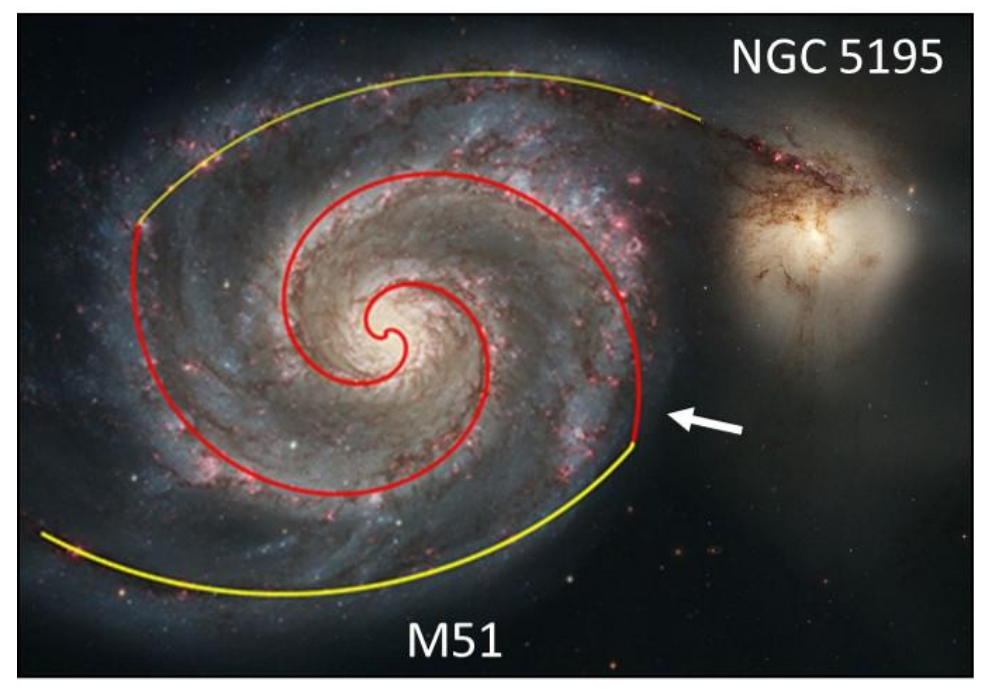

Figure 7: Galaxy M51 and spiral pattern simulation by ROTASE model, Euler(13,0,0).

Based on the proposed ROTASE model, the formation of the two distinguishable sections of each spiral arm is due to the central SMBH in M51 emitting the X-matter with two very different emission behaviors as shown in Figure 7. From the galactic bar rotation angle time $485^{\circ}$ to $610^{\circ}$ in the past from now, it emitted the $\mathrm{X}$-matter to produce the yellow line spiral arm sections with parameter $\rho$ following the equation:

$$
\rho=0.00088 *(\theta-535)^{2}+11.704
$$

However, from the galactic bar rotation angle time $485^{\circ}$ in the past to now, the central SMBH has been emitting the $\mathrm{X}$ matter to produce the red line spiral arm sections with parameter $\rho$ following the equation:

$$
\rho=1.1 *(1+0.024 * \theta)
$$

The change of the X-matter emission behavior by its central SMBH was clearly described previously [16]. The equations (12) and (13) indicate that the X-matter emission velocity in the past gradually decreased, then, at rotation angle $535^{\circ}$ time from current, the $\mathrm{X}$-matter emission increased for short period of time (about $50^{\circ}$ rotation time), then, it has been steadily decreasing up to today [16]. We will see more cases with the sudden change of the X-matter emission behaviors by central SMBHs in later sections. The trail effect can be clearly seen in this image.

M51 has a companion galaxy NGC 5195 shown in the upper right. In the tidal interaction model, the spiral arms are the result of tidally induced kinematic density 'waves' or density patterns, which wind up slowly over time [14]. However, this tidal induced mechanism for the formation of spiral arms with perfect central symmetry is realistically implausible, because the intensity of such tidal interaction by the gravitational force between the two cosmic scaled large objects is extremely (central) unsymmetrical related to the M51 center. The top yellow arm of M51 is extended to its companion galaxy NGC 5195 by the strong gravitational force and the morphology of the lower arm 
section pointed by the white arrow is changed by the NGC 5195 with strong gravitational force, the other side of the spiral arm is not affected due to distance from the NGC 5195. The two galaxies are constantly moving, the distance between the two galaxies is constantly changing, realistically, it is hard to believe that such unsymmetrical interaction can produce the spiral arms with perfect central symmetry in the M51 galaxy. It is more reasonable to believe that the spiral structure of the M51 was formed with perfect central symmetry long before the two galaxies approached each other, so the unsymmetrical tidal interaction between the two galaxies damages the central symmetry.

\section{Galaxy MCG+00-04-051 with spiral arms not bound to the ends of galactic bar}

The galaxy MCG+00-04-051 is a very interesting galaxy with its spiral arms apparently disconnected from the galactic bar ends as shown in the Figure 8a. This galaxy was studied in detail previously as shown in Figure 8b with simulation by the ROTASE model [17], one of the spiral arms is still connected to the galactic bar end with a weak but clearly visible spiral arm AC marked by the red dash line. The unusual pattern was clearly explained by the ROTASE model, which is due to the complete termination of the X-matter emission at one side of the SMBH and but dramatical decrease of the X-matter emission at other side of SMBH. In a previous study by Seigar and James [23], the gaps between the spiral arms and the galactic bar ends were believed due to the arms moving (rotating) ahead of the bar. The weak spiral section AC proves that the galactic bar rotates ahead of spiral arms not behind of spiral arms. Such pattern fully matches the prediction by ROTASE model as described in Figure 20 of reference [16]. Although, the distance from spiral arm end D to galactic bar end $\mathrm{C}$ is much smaller than the distance from $\mathrm{D}$ to galactic bar end B, and gives a false impression that D is ahead of $\mathrm{C}$, such false impression will be certainly accepted without question if the weak AC spiral section does not exist. In reality, all other models cannot explain the morphology of this galaxy. Based on the manifold model, the spiral arms started at the L1 and L2 Lagrangian points which are located at the straight line of galactic bar and close to the ends of the galactic bar [10], however, in this galaxy, the "starting" points A and D of the arms are not at the L1 and L2 points, and do not match this model; how does this manifold model explain the weak but still visible arm section AC? Density Wave Theory cannot explain this phenomenon either.
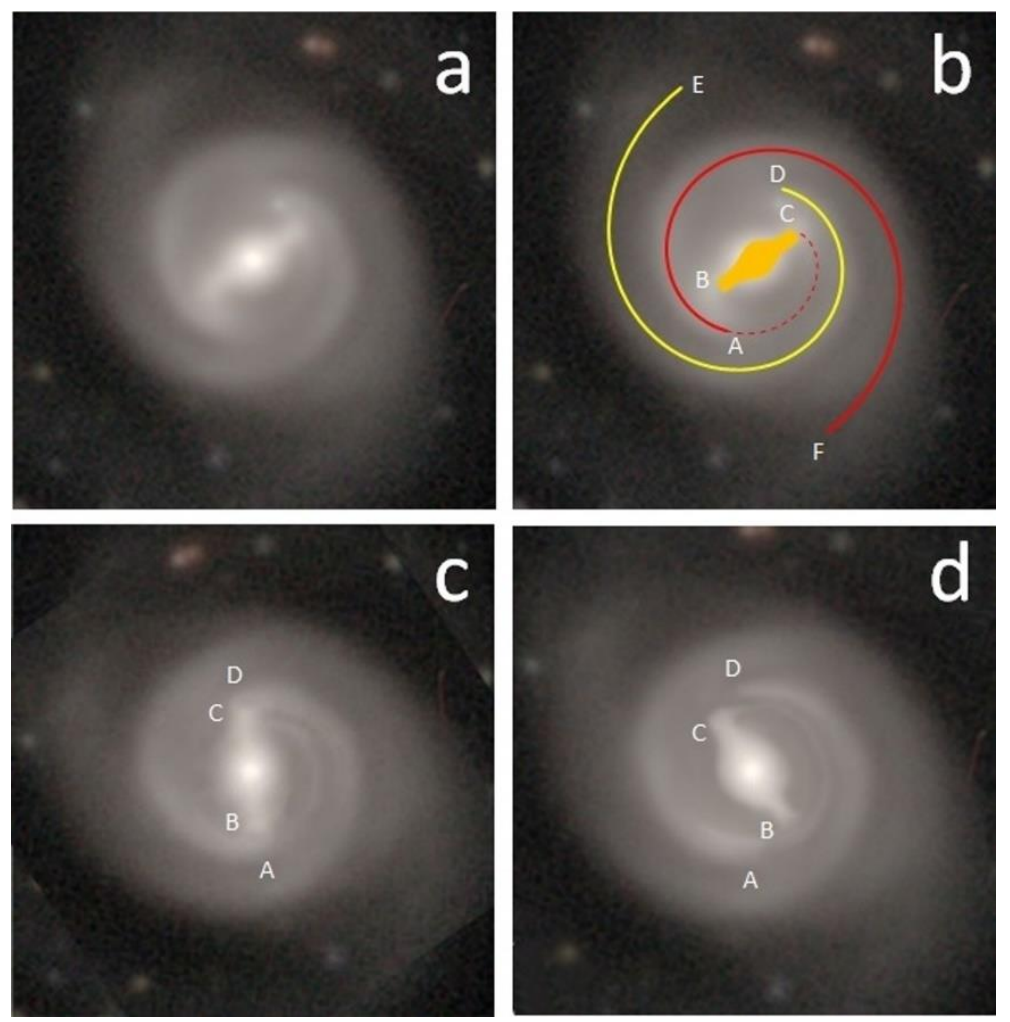

Figure 8: a: the original image of galaxy MCG+00-04-051; b: Simulation by the ROTASE model, Euler (-57, 0, 0); c: possible pattern in near future after $30^{\circ}$ to $40^{\circ}$ bar rotation; d: possible pattern after additional $10^{\circ}$ to $13^{\circ}$ bar rotation.

The ROTASE model predicts that if the current trend continues, the gap between $\mathrm{A}$ and $\mathrm{B}$ as well as the gap between $C$ and $D$ will gradually decrease with time. In about $30^{\circ}$ to $40^{\circ}$ of galactic bar rotation, the bar end $\mathrm{C}$ will catch up the end D of spiral arm and the bar end B will catch up the end A of spiral arm. The ends of the bar and the ends of spiral arms will line up in a straight-line A-B-C-D, the spiral arm end A will look like it could be connected to the bar end B, the spiral arm end D will look like it could be connected to the bar end $\mathrm{C}$ if the gap between $\mathrm{A}$ and $\mathrm{B}$ as well as the gap between $C$ and $D$ are close enough. The image of the galaxy will look like a "regular" spiral galaxy as shown in Figure 8c, which will give a false impression, the weak section AC may still be seen or become weaker, may disappear. In this scenario, without referring to the ROTASE model, it would be laughed at if someone says 
that the spiral arm AF is originated from bar end $\mathrm{C}$ and the spiral arm DE is originated from bar end B. When the bar continues to rotate, the galactic bar will overpass the A and $\mathrm{D}$, for another $10^{\circ}$ to $15^{\circ}$ rotation, the galactic bar will be ahead of the ends of spiral arms as illustrated in Figure 8d. However, if one believes that the spiral arms rotate ahead of the galactic bar in the original image Figure 8a, then, it will predict that the gap between $A$ and $B$ and the gap between $\mathrm{C}$ and $\mathrm{D}$ will increase with time for the near future. Readers may make their own judgements for which prediction is reasonable. The author feels confident that patterns shown in Figure 8c and Figure 8d will certainly occur in the future if no dramatically exceptional event happens. If we use the galactic bar rotation velocity of Milky Way as a reference, $30^{\circ}$ to $40^{\circ}$ rotation takes about
10 to 13 million years. 10 to 13 million years is a fleeting moment in the evolution time scale of the universe, but is too long compared to our human history. Such predictions are realistically not observable in the foreseeable future. The galaxies with patterns similar to Figure $8 \mathrm{c}$ and Figure $8 \mathrm{~d}$ should exist in the universe, which may be already in the database or to be detected in the future.

The galaxy NGC 4548 has the same morphology as the MCG+00-04-051. It has an apparent broken connection of the spiral arms from the bar ends in an image with low quality shown in Figure 9 left, but the image with high quality clearly shows that the arm A is connected to the bar end with a weak and still visible spiral arm. The arm B is not connected to the other bar end as shown in Figure 9 right, and it was studied in detail in the reference [17].
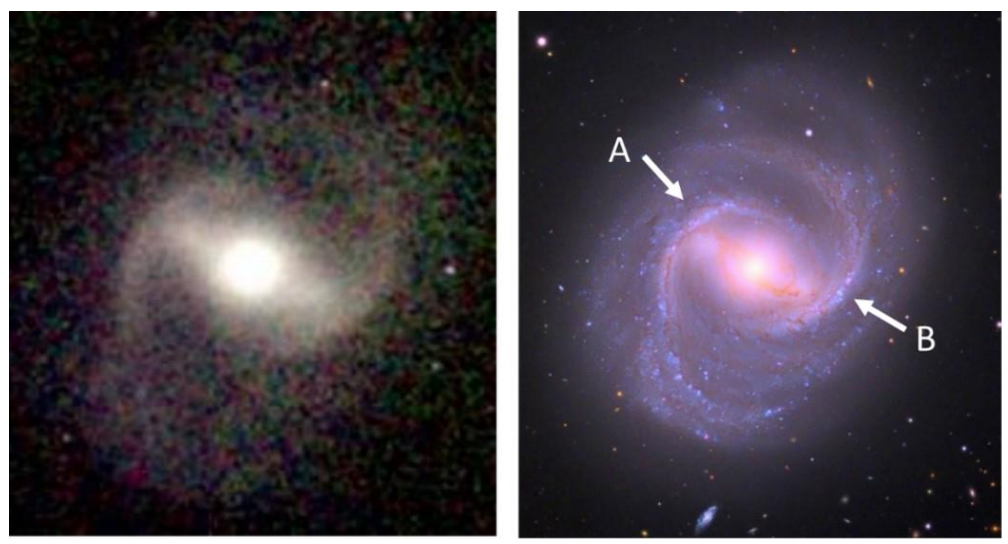

Figure 9: Left: the image of NGC 4548 with low quality; right: image of NGC 4548 with high quality.

\section{Galaxies with rings}

Galaxies with spiral rings are very popular and can be classified into four major types based on the significant difference of the morphologies. Type I: single ring; type II: 8-shaped double ring; type III: 8-shaped double ring wrapped by a larger outer ring; type IV: single ring without spiral and bar. All four types of ring galaxies can be described by the ROTASE model.

\subsection{Type I: Galaxies with a single ring pattern}

The image of the galaxy CGCG 119-82 on the left of Figure 10 shows almost a perfect round ring with a strong bar without the visible spiral structure. It may be classified as a barred lenticular galaxy which contains a large disc without spiral arms. It is a face-on image and can be simulated nicely by the ROTASE model with $\rho=0.1$ as shown in Figure 10 right. Furthermore, the simulation with other small $\rho$ values $(<0.15)$ will also show the same result because the ring and the bar ends are close enough and merged by the blurry optical effect. Based on the ROTASE model, such a pattern is the result of weak X-matter emission (low $\rho$ value means low $\mathrm{X}$-matter emission velocity). It still has the spiral structure which is hidden under what looks like a combined ring and bar ends. Because the space between the ring and bar ends is too short to be seen, the direction of the galactic rotation is unknown from the image. The spiral structure of the spiral ring galaxies will increase with the value of $\rho$ and will be more visible as shown in Figure 11.
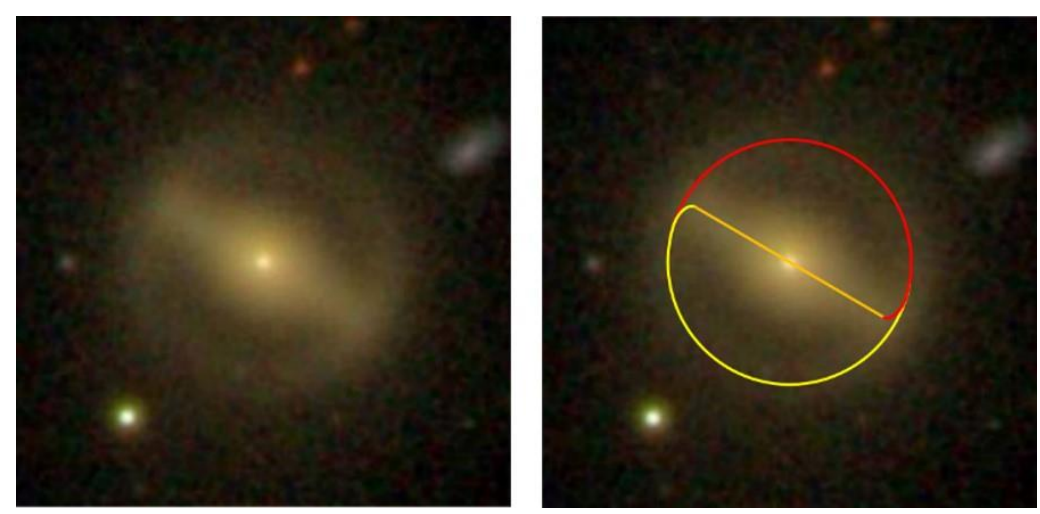

Figure 10: Left: the original image of CGCG 119-82; right: the simulation by ROTASE model, $\rho=0.1$, Euler $(60,0,0)$. 

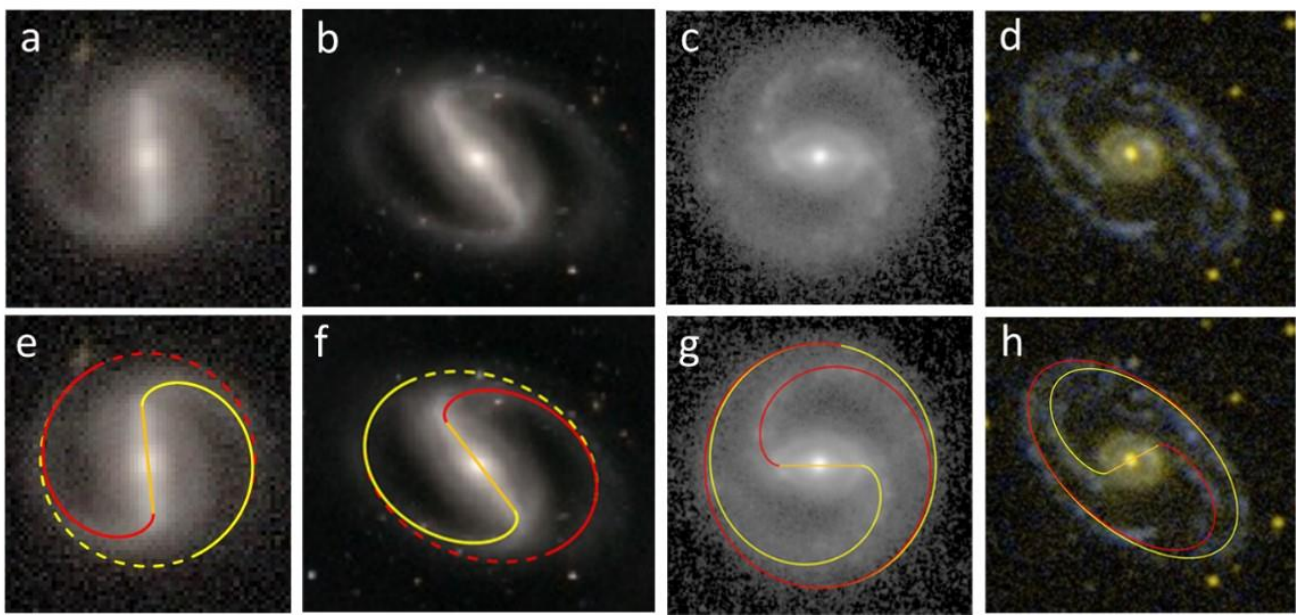

Figure 11: First row are the 4 original images of galaxies with single spiral-ring patterns: a: unknown-name ring 1 from reference [24], b: unknown-name ring 2 from reference [24], c: ESO 325-28; d: NGC 2273. Second row are the images with simulations by the ROTASE model for each related original galaxies in the first row, e: $\rho=0.5$, Euler $(6,0,0)$; f: $\rho$ $=0.5$, Euler(-25,40, 55); g: ESO 325-28, $\rho=0.65$; h: NGC 2273, $\rho=0.6$, Euler $(-43,57,80)$.

The images of unknown-name ring 1 and ring 2 galaxies in Figure 11a and 11b are duplicated from reference [24]. The original images show tightly wound open (not closed) spiral patterns which may be classified as SBa type galaxies. However, in Figure 11a and 11b, when the visible spiral arms are simulated by the solid red and yellow lines with constant parameter $\rho=0.5$, this results in a single spiral-ring pattern if the visible arms are extended longer shown by the dashed red and yellow lines. Therefore, the two galaxies are concluded to have a truncated single spiral-ring pattern due to the decay of the luminosity along the arm line from the end of galactic bars. The density and the age of the stars along the circle of spiral arm do not cause such decay in luminosity, because the density and age of the stars in the circle are more or less the same. The density wave theory explains the ring formation as resonance of a density wave in this radius, but such resonance cannot explain the gradual decrease of the luminosity along the arm lines. The ROTASE model seems to have a more reasonable explanation that the gradual decrease of the luminosity along the arm lines is only caused by the gradual decrease of hydrogen conversion by the X-matter along the circle of spiral arm lines or "aging" of the arms. The ESO 325-28 galaxy shows a nice face-on single spiral-ring pattern and the NGC 2273 shows a nice single spiral-ring pattern with a large inclination angle. Both the ESO 325-28 and NGC 2273 show a significant spiral structure. The radii of the rings will follow equation (4) and will increase non-linearly with the $\rho$ values. Figure 12 shows the single spiral-ring patterns with four different $\rho$ values; when $\rho=1$, the radius is infinite, i.e., no ring. However, in reality, galaxies with only less than 2 windings of spiral arms are observed. Most galaxies have half to one loop winding as marked by " $\mathrm{X}$ " in Figure 12 for ring pattern with $\rho=0.8$. The spiral arms beyond " $X$ " are not visible due to the fade away with the decrease of the hydrogen conversion from the X-matter (the amount of X-matter decreases). These galaxies will have truncated spiral-ring patterns, and will be classified as $\mathrm{SBa}$ or $\mathrm{SBb}$ patterns in Hubble sequence. In the classical theory of the stellar dynamics of spiral galaxies, the radius of the spiral arms (the size of the galaxies) may be limited by $1 / 4$ Lindblad resonance [25]. Beyond $1 / 4$ Lindblad resonance, the disc is not stable, and the spiral arms will not be stable also. However, such a limitation cannot explain the gradual decay of luminosity along the circular line of the spiral arms of the spiral ring galaxies like Figure 11a and $11 \mathrm{~b}$.
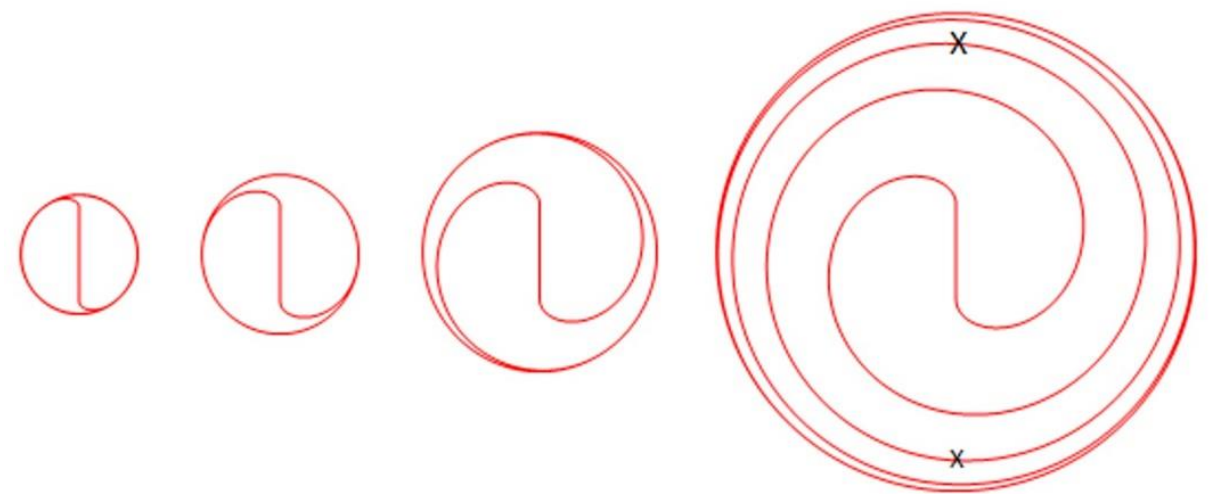

$$
\rho=0.2 \quad \rho=0.4
$$

$$
\rho=0.8
$$

Figure 12: the ring size of the galaxies changes with the parameter $\rho$. 


\subsection{Type II: Galaxies with 8-shaped double ring patterns}

Figure 12 shows 4 galaxies with 8 -shaped double ring patterns, NGC 7098, UGC12646, NGC 2665 and SDSS J015701.50-001644.4. The upper row are the original images and the lower row are the simulations by the ROTASE model corresponding to their original images

above. The parameter $\rho$ of this type double ring patterns follows the general Gaussian equation:

$$
\rho=\rho_{0} * \exp \left(-\mathrm{k} *\left(\theta-\theta_{\mathrm{p}}\right)^{2}\right)
$$

The $\rho_{0}$ is the maximum value of $\rho$; $\mathrm{k}$ is a constant controlling the width of the Gaussian profile; $\theta_{\mathrm{p}}$ is the angle (time) in the past from now, and at $\theta_{\mathrm{p}}$ the $\mathrm{X}$-matter has the peak emission.
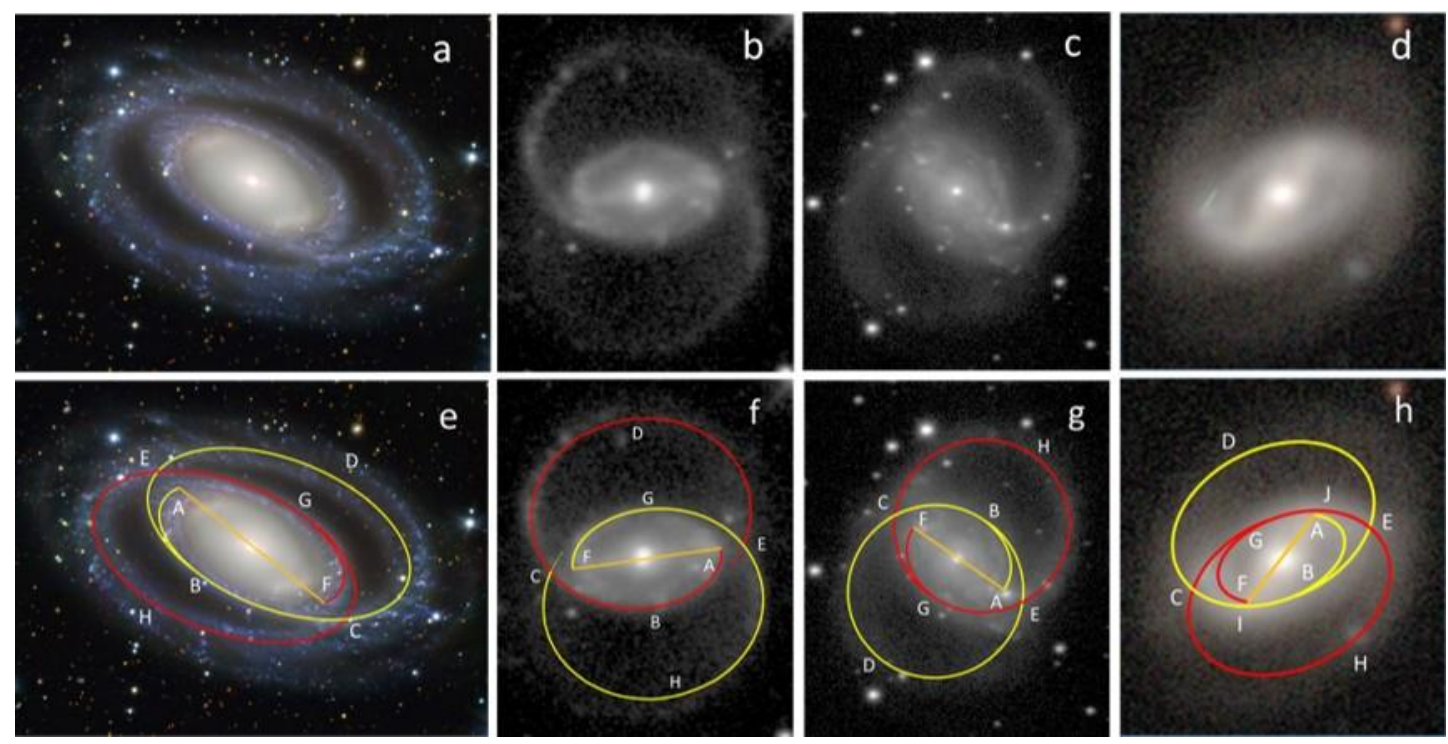

Figure 13: four galaxies with 8-shaped double ring pattern; Top row is the original images, a: NGC 7098, b: UGC12646, c: NGC 2665, d: SDSS J015701.50-001644.4. Lower row is the simulations for each original image above. e: NGC 7098, Euler (25,65,25); f: UGC12646, Euler (8,59,0); g: NGC2665, Euler (35,35,0); h: SDSS J015701.50-001644.4, Euler $(-22,-55,45)$.

The simulation parameters of four 8-shaped double ring galaxies are summarized in the Table 1 below:

Table 1: Simulation parameters for the 8-shaped ring galaxies listed in Figure 13.

\begin{tabular}{|c|c|c|c|}
\hline Name of galaxies & $\rho_{0}$ & $\mathrm{k}$ & $\theta_{\mathrm{p}}$ \\
\hline NGC 7098 & 0.55 & 0.0001 & 360 \\
\hline UGC 12646 & 0.655 & 0.00013 & 360 \\
\hline NGC 2665 & 0.65 & 0.0001 & 360 \\
\hline SDSS J015701.50-001644.4 & 0.65 & 0.0001 & 410 \\
\hline
\end{tabular}

The morphology of 8-shaped double ring pattern looks like a small inner ring wrapped with a larger outer ring. However, with careful inspection, one can find that the outer ring and inner ring are connected at the ends of galactic bar area marked by $\mathrm{C}$ and $\mathrm{E}$ in Figure $13 \mathrm{e}$ and Figure 13f, the connection sections have smooth spiral characteristics with central symmetry. Buta noticed this interesting phenomenon and marked "The outer ring is complicated by the emergence of spiral structure from its minor axis region, and I believe what we are seeing in this case is an R1R'2 morphology where the R'2 component is not as fully developed as in NGC 1079 and 3081" [26]. The ROTASE model reveals that the double rings are actually made of two identical rings, each ring is made of a half inner ring and a half outer ring; and each ring crosses other ring twice with chain-link style. and the sequential decrease of luminosity of the spiral ring along the arm lines is due to the sequential decrease of X-matter during its motion which causes the decrease of hydrogens converted from the Xmatter, the distance and the ages of the stars in the spiral arms have little or no effect on the sequential decrease of the luminosity. The chain-link arm crossing was first named in the previous paper to describe the local detail of the spiral arm crossing more accurately and how the outer ring is developed [17]. The detailed characteristics of the 8shaped double ring galaxies can only be explained by the ROTASE model at the moment, all other available hypotheses cannot explain such phenomena.

The simulation parameters in Table 1 indicates that the galaxies NGC 7098, UGC 12646 and NGC 2665 were developed at the same time period and the peak emission of the X-matter by the central $\mathrm{SMBH}$ was at $360^{\circ}$ rotation time in the past from now.

However, the galaxy SDSS J015701.50-001644.4 was developed $50^{\circ}$ rotation time earlier. The peak emission of the X-matter by the $\mathrm{SMBH}$ is at $410^{\circ}$ of galactic bar rotation time in the past from now, which is about $50^{\circ}$ rotation time ahead of NGC 7098, UGC 12646 and the NGC 2665. The minor axis is about $50^{\circ}$ from the galactic bar. The major axis of outer ring is about $40^{\circ}$ from the galactic bar. It cannot be classified by Buta's classification as either R1' subclass (as defined as $180^{\circ}$ winding relative to the bar axis) or R2' subclass (as defined as $270^{\circ}$ winding relative to the bar axis) [27]. It is instead between $\mathrm{R} 1$ ' and R2', but not exactly a mix of the R1' and R2' either. The arm crossing points $\mathrm{C}$ and $\mathrm{E}$ are not located at the Lagrangian points L1 and L2 or other Lagrangian points L4 and L5 in the Manifold model. The dark spaces of this galaxy are not located at the L4 and L5 Lagrangian points 
which does not match Buta's interpretation [28]. The image of this galaxy shows that the luminosity sequentially decreases along the arm lines with outer rings almost fading away. If the galaxy continues evolving with the same trend, the outer ring will disappear in the future and become a galaxy with its morphology similar to CGCG 119-82 above. The ROTASE model is able to produce a pattern that fully matches the current morphology. Any successful theory for the formation of spiral arms of galaxies must be able to clearly explain why the arm crossing points do not align with the galactic bar axis, nor perpendicular to the galactic bar axis. From the ROTASE model, it is just a regular chain-link 8-shaped double ring galaxy with sequential decrease of luminosity and quality, the arm crossing points can be located at any places on the rings depending on when the peak emission occurs in its history.

\subsection{Galaxy J102942.99-022704.0 with 8-shaped unequal double rings}

The original image of the galaxy J102942.99-022704.0 is shown in Figure 14 left, the quality of the image is not high, but it still clearly shows the 8-shaped double ring pattern with unequal double rings, the two rings are very unsymmetrical, the south ring (yellow) is much narrower than the north ring (red).

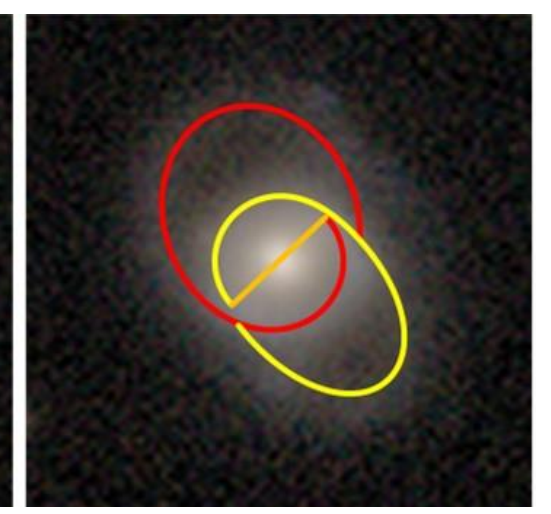

Figure 14: Galaxy J102942.99-022704.0, left: original image, right: the simulation by ROTASE model, Euler (-47,0,0,).

The unusual pattern can be nicely simulated by the ROTASE model with the following parameter $\rho$ equations:

$$
\rho=0.6 * \exp \left(-0.0001 *(\theta-380)^{2}\right)
$$

for the north ring (red), and

$$
\rho=0.6 * \exp \left(-0.00025 *(\theta-360)^{2}\right)
$$

for the south ring (yellow). Apparently, the X-matter emissions at the two sides of the SMBH are not equal which caused such unequal 8 -shaped double ring pattern. It is impossible that this galaxy can be formed by density wave mechanism. The ring crossing points are not central symmetric and are not located at any Lagrangian points. Any successful theory for the formation of the spiral arms must be able to clearly explain how this unequal ring pattern is formed.

\subsection{Type III: Galaxies with 8-shaped double ring wrapped with a larger outer ring}

This type of ring patterns is much more complicated than type II galaxies. Figure 15 shows 4 galaxies with such complicated ring patterns. The top row is the galaxy NGC 1079 , it was studied by the ROTASE model in previous paper [17], the left is the original image, and the middle is the pattern profile depicted by Buta and the spiral ring pattern is classified as R1R2' mix pattern by Buta [28]. The right is the simulation by the ROTASE model with the parameter $\rho$ following the Gaussian equation:

$$
\rho=0.6 * \exp \left(-0.000015 *(\theta-450)^{2}\right)
$$

Its major axis lines up with the galactic bar and the minor axis is perpendicular to the galactic bar. The peak emission of the X-matter by $\mathrm{SMBH}$ is at $450^{\circ}$ of galactic bar rotation time in the past from now. The simulation shows that the complicated ring pattern is actually made of two identical rings. The red line arm is developed in the sequence of ABCDEFCG, the time sequence of the spiral arm development is actually reversed from $G$ to A, i.e., $G$ is developed first. The red line arm crosses the yellow arm at $\mathrm{D}$ and $\mathrm{F}$ and crosses itself at $\mathrm{C}$. So, the complicated ring pattern is actually made of two identical rings and each ring is made of a half outer ring and multi section inner rings, each ring crosses other ring twice and crosses itself once. The ROTASE model is able to produce the spiral pattern which matches the image well and explain how such pattern is developed. The Figure 15 middle row is the galaxy 3081 , left is the original image, middle is the spiral ring pattern profile depicted by Buta [29] and the right is the simulation of the Disk-subtracted B-band image [30] by ROTASE model with parameter $\rho$ following the Gaussian equation:

$$
\rho=0.6 * \exp \left(-0.000015 *(\theta-450)^{2}\right.
$$

The characteristics of the pattern should be the same as the NGC 1079, will not be repeated here. The Figure 15 bottom row are the depicted pattern profiles of ES 509-98 and ESO 507-16 by Buta [26], they all have R1R2' mixed pattern classified by Buta. The ESO-507-16 has the same pattern as the NGC 3081. But the ESO 509-98 seems having irregular R1R2' pattern, the profile does not have central symmetry, the major axis does not line up with the galactic bar nor perpendicular to the galactic bar. The arm cross-points do not seem to line up with the major axis nor the minor axis. The ROTASE model would interpret this as 
the X-matter emissions at two sides of the central SMBH are not equal and the parameter $\rho$ may not exactly follow the Gaussian equation (14). Any successful theory for the formation of spiral arms must be able to clearly explain the formation of type III double ring pattern.
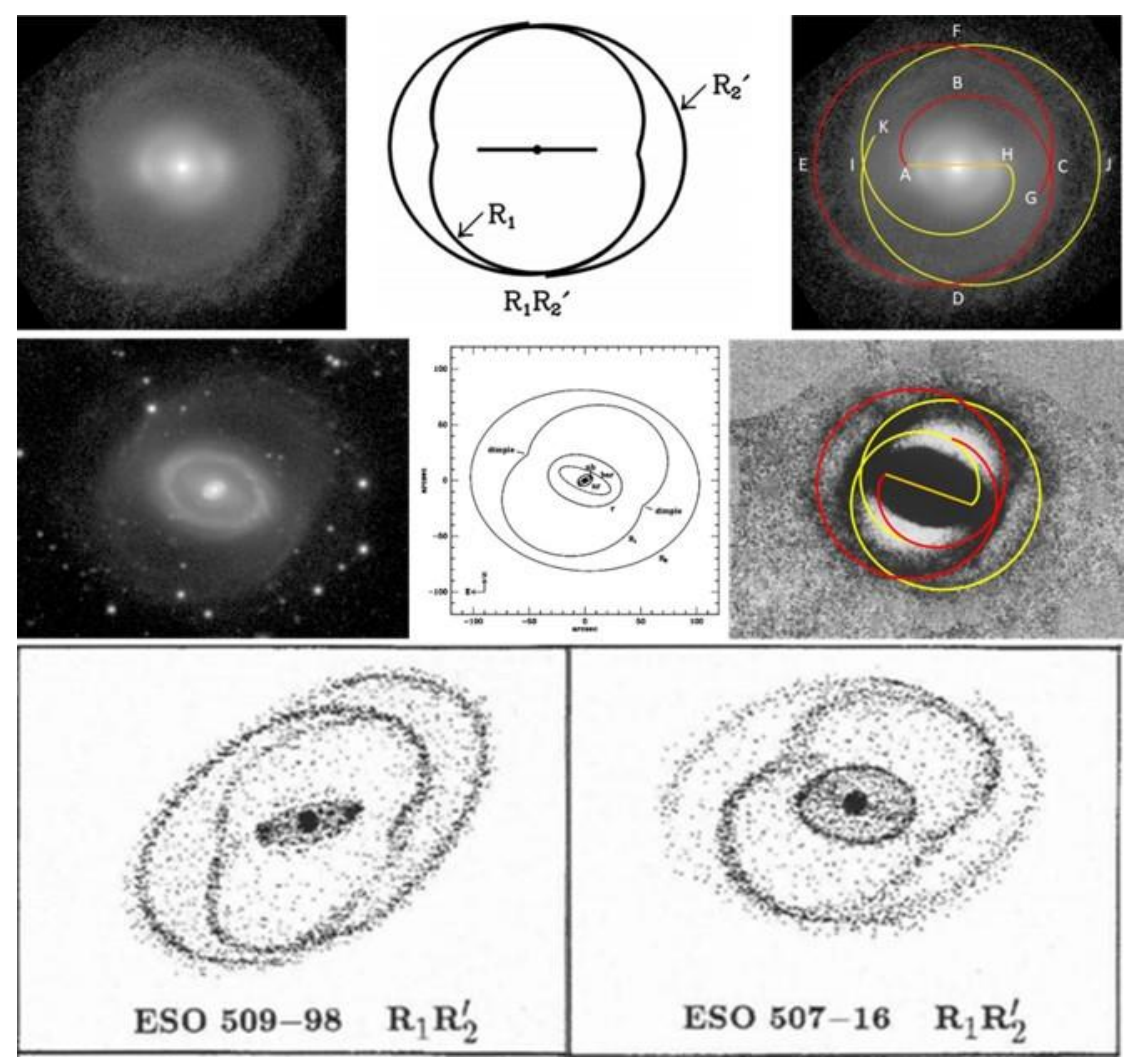

Figure 15: type III ring pattern with 8-shaped inner ring wrapped by a larger outer ring.

Top: NGC 1079, left: original image, middle: depicted pattern profile [28], right: simulation.

Middle: NGC 3081, left: original image, middle: depicted pattern profile [30], right: simulation, Euler (-20,5,40).

Bottom: pattern profiles depicted by Buta [26], left: ESO 509-98, right: ESO 507-16

\subsection{Type IV: Galaxies with pure ring patterns without spirals and galactic bars}

Hoag's object is one of the most fascinating objects in the sky as shown in Figure 16 left. It has an almost perfect ring dominated by bright blue stars, while near the center lies a ball with much redder stars that are likely much older. Between the bugle and the ring is a gap that appears almost completely dark. The Figure 16 right is the galaxy ISI961159, which looks like a twin of Hoag's object. We have to admire the nature's superpower to create such beautiful artworks.
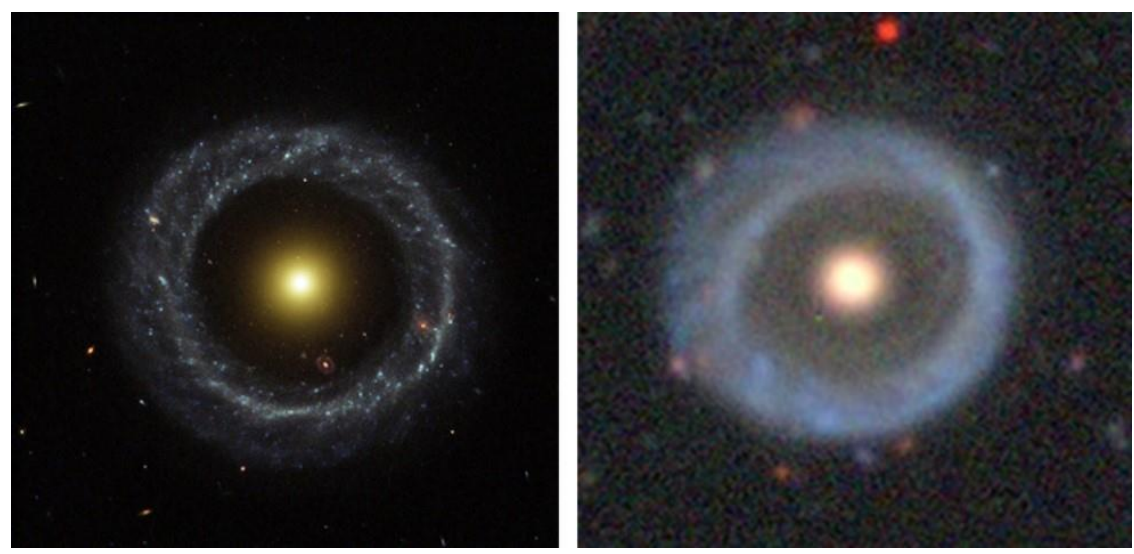

Figure 16: left: Hoag's object; right: ISI961159

The mechanism for the formation of the Hoag's object is still not clear. Hoag himself proposed that it is the gravitationally lensed image of a background galaxy [31]; the other theory proposed that the rotation speed of the galactic bar gradually increases to a certain point that the bar structure is not stable and breaks down to spirally spinout, the density waves are migrated out into a ring-structure which may be called a resonance ring. Galactic materials will accumulate in the ring area by pressure and gravitational force to promote new star formation, and a 
spherical bugle is leftover at the center with redder and older stars [32]. The possible collision between galaxies may produce this ring structure also [33, 34]. Schweizer and et. al., studied Hoag's object with photometric and spectroscopic observations, and their findings ruled out all those three hypotheses. They proposed a new hypothesis of accretion with mass transfer between galaxies, and this accretion event took place at about 2-3 Gys ago [35].

However, the ROTASE model will give a different explanation for the formation of Hoag's object. From the galaxies MCG+00-04-051 and M51 above, we learned that the X-matter emission by the central SMBH can change at any time in any format. For galaxies with a spiral ring pattern like CGCG 119-82, ESO 325-28, and NGC 2273 above, if the $\mathrm{X}$-matter emission is completely terminated by the central SMBH, the X-matter emitted before the termination will continue to spiral outwardly into the ring and the extra hydrogens in the bar will be depleted. The spirals will disappear, and the bar will gradually shrink from a bar to a US football-like egg shape. The galactic bar will further shrink and eventually will become a spherical ball bugle at the center with redder and older stars due to depletion of the extra hydrogens; the X-matter in the ring will continue to be converted to hydrogens to refuel the local stars and promote new star formation. Therefore, only the ring and the central bugle will be visible like the current Hoag's object. The explanation by ROTASE model is supported by the morphologies of NGC 1291 and NGC 6028 shown in Figure 17.

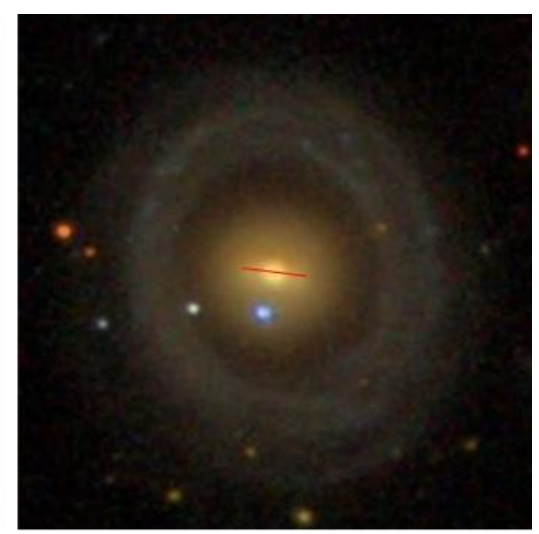

Figure 17: Left: NGC 1291; right: NGC 6028

Both NGC 1291 and NGC 6028 are barred galaxies without spiral structures. However, with a rough visual estimate, the length of the bar is about half of diameter of the ring for NGC 1291, and the length of bar is about a quarter of the diameter of the ring for the NGC 6028, as marked by red lines in the images. This indicates that the bar of NGC 1291 is in the early stage of bar shrinking. It has already shrunk to an egg-shaped bar, and the spirals have disappeared. The galactic bar of NGC 6028 is in late stage of shrinking. It will disappear soon and become a true Hoag's object galaxy. Therefore, both galaxies are excellent examples of intermediate galaxies for the transition from spiral ring galaxies to Hoag's objects and solid support for the ROTASE model.
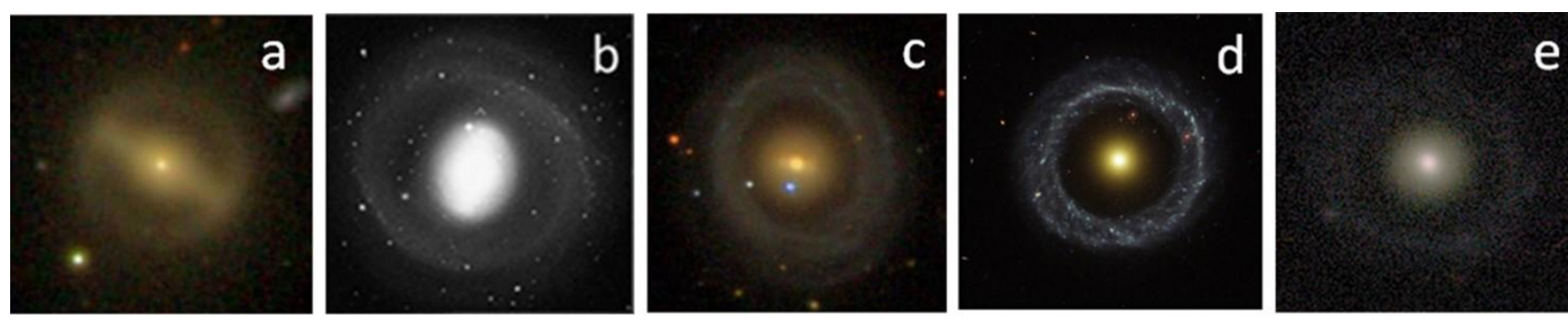

Figure 18: the possible evolution sequence of the Hoag's object from a to e. a: CGCG 119-82, b: NGC 1291, c: NGC 6028, d: Hoag's object, e: J084119.22+271036.2

If the galaxy evolves with the same trend, i.e., the black hole will not resume the $\mathrm{X}$-matter emission in the near future, the ring and bugle will be dimmer and dimmer like the galaxy J084119.22+271036.2 shown in Figure 18e, in which the outer ring is barely visible. It will be eventually invisible due to complete depletion of the extra hydrogens and temperature decrease of the bugle, but the galaxy still exists like an invisible ghost wandering in the universe. The universe should have a lot of dark/invisible galaxies wandering around which may collide/merge with other galaxies. Figure 18 shows the possible evolution sequence of the Hoag's object from Figure 18a to Figure 18e. The formation of the Hoag's object is just a natural consequence predicted by the ROTASE model when the X-matter emission is terminated for a galaxy with the single spiralring pattern. Any successful theory for the formation of spiral arms must be able to clearly explain how the Hoag's object is formed.

\section{Galaxy NGC 4618 with one spiral arm}

The results of the galaxy MCG+00-04-051 and NGC 4548 show that the X-matter emission behavior of the central SMBH can change in any format and at any time. 
The emissions at the two sides of the SMBH can be also unequal. It will be naturally concluded by ROTASE model that a galaxy may have only one arm if one side has weak or no X-matter emission, but other side has strong X-matter emission. The famous NGC 4618 is one of these one-arm galaxies. The luminosity sequentially decreases along the arm line shown in Figure 19 left, the arm looks a normal spiral arm like other regular spiral arms without no any specialty. Kaczmarek and Wilcots studied in detail about NGC 4618 and ruled out the possible mechanism for the formation of the one-arm galaxy by the gravitational interaction between NGC 4618 and NGC 4625 [36]. From Figure 6 , the spiral arms of the galaxy have to be central symmetric by the density wave theory, so, the one-arm galaxy cannot be formed by the density wave mechanism, it cannot be explained by the manifold theory either. Therefore, a one-arm galaxy must be formed by an internal process that is still unclear so far. The one arm spiral pattern of NGC 4618 can be simulated nicely shown in the Figure 19 middle by the following parameter $\rho$ equation with $505^{\circ}$ rotation angle:

$$
\rho=0.65 * \exp \left(-0.000025 *(\theta-415)^{2}\right)
$$
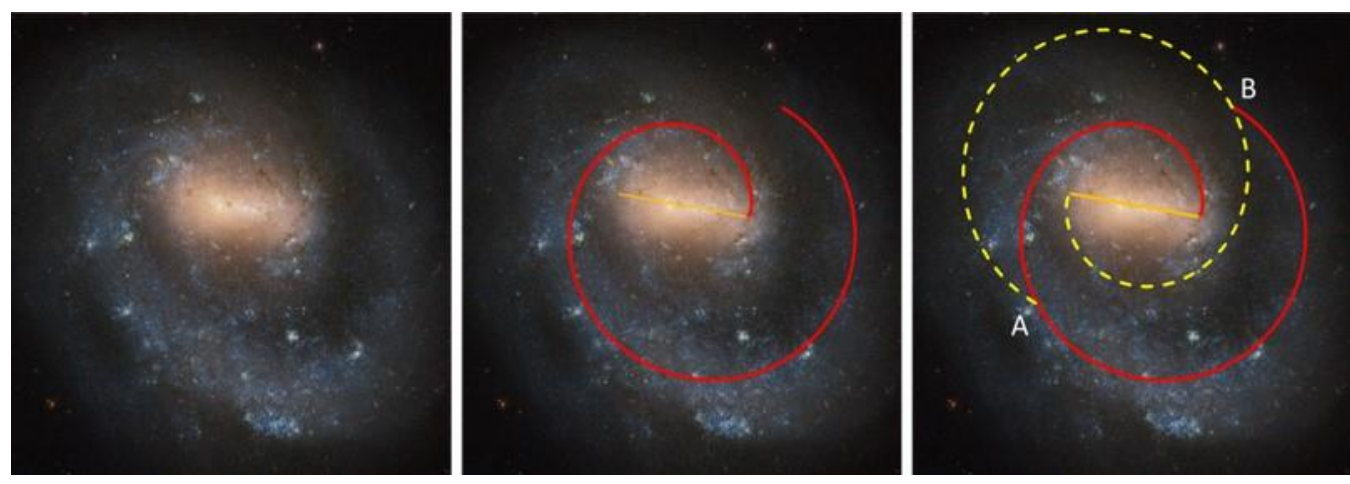

Figure 19: NGC 4618 with one spiral arm and simulation, Euler(10,0,0).

The parameter $\rho$ equation (19) matches the general parameter equation (14) for ring galaxies. The one spiral arm is a truncated ring pattern, and the NGC 4618 galaxy would be a perfect 8-shaped chain-link double ring galaxy if it had the other ring as shown in Figure 19 right with yellow dash line. The cross points would be at locations A and B. Due to the symmetry of A and B with respect to the galactic center, the density and the ages of stars as well as the physical environment in A and B areas should be more or less the same, but the luminosity of area A is much stronger than area B. This stronger luminosity in area A reflects brighter stars and more new star-forming activities, which requires much more hydrogens. Why does the area A have much more hydrogens than the area B? The ROTASE model seems the only one to give a logical and natural explanation at the moment. The pattern of the NGC 4618 perfectly matches the ROTASE model and is a good solid support for the model. Any successful theory for the formation of spiral arms must be able to clearly explain the formation of this one-arm galaxy.

\section{Possible description of the evolution of the spiral pattern by the ROTASE model}

Based on the ROTASE model, the spirals of the galaxies are developed from the inside out in time sequence. This provides a possibility to project the possible spiral pattern evolution of the galaxies as illustrated with the galaxies UGC 6093 and M51.

\subsection{The possible spiral pattern evolution of the galaxy UGC 6093}

The spiral pattern of UGC 6093 can be nicely simulated shown in Figure 20. The image of the galaxy clearly shows three distinguishable development stages by yellow, red and blue lines, respectively. The blue line section is simulated by the following parameter $\rho$ equation for the galactic bar rotation angle $0^{\circ}$ to $270^{\circ}$ :

$$
\rho=0.85 * \exp \left(-0.000015 *(\theta-500)^{2}\right)
$$

The red line section is simulated by the following parameter $\rho$ equation for the galactic bar rotation angle $271^{\circ}$ to $470^{\circ}$ :

$$
\rho=0.384+0.0012 *(\theta-270)
$$

And the yellow line section is simulated by the following parameter $\rho$ equation for the galactic bar rotation angle $471^{\circ}$ to $575^{\circ}$ :

$$
\rho=0.624 * \exp (0.002 *(\theta-470))
$$




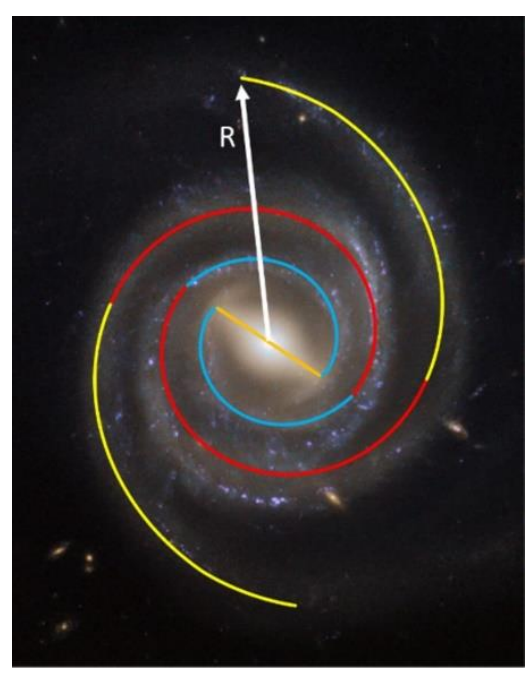

Figure 20: Three section simulations for $\operatorname{UGC} 6093$, Euler $(57,0,0)$

Please remember that the $\rho$ is the ratio of the X-matter emission velocity out of the confined route over the disc flat rotation velocity. Therefore, a change in $\rho$ with time means the X-matter emission velocity changes with time. If $\rho$ increases with backward time, this means that the X-matter emission was larger in the past. Here, the rotation angle $\theta$ is equivalent to the evolution time scale. Figure 21 shows the parameter $\rho$ change with time from past to current time. The $\rho$ has been continuously decreasing from $575^{\circ}$ galactic bar rotation time ago to now. This means that the X-matter emission by the central SMBH has been continuously decreasing during this time period, and the central SMBH may gradually evolve to a new stage.

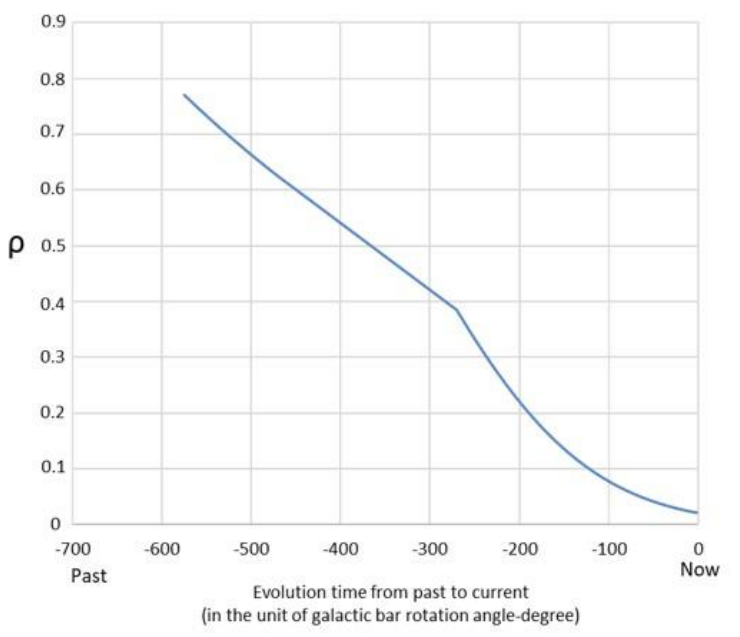

Figure 21: Parameter $\rho$ change with evolution time of the galaxy UGC 6093.

The $\mathrm{R}$ in Figure 20 is the radius of the maximum visible spiral arm in this image and will be used as a reference size of the galaxy during the illustration of its spiral pattern evolution. The two opposite entirely visible arms show almost perfect winding symmetry with respect to the galactic center in each different development stage (yellow, red and blue). This continuous change of the morphology with perfect central symmetry must have happened internally, because if such change was caused by external impacts (like other galaxies or objects), then there would have to be two forces that were separate, simultaneous, opposite, and identical in intensity and time. It is likely impossible that such perfectly collaborative impacts would happen simultaneously. According to the Density Wave Theory, the spiral arms are density waves which are initiated by instability $[14,37]$. If the instability happened in the non-central locations, could the instability produce such perfectly centrally-symmetric long arms with nice winding in nature? Most likely, the possibility is zero. It is much more reasonable to believe that the arms were produced at the ends of galactic bar and swirled outwardly as in the ROTASE model proposed. The current spiral pattern of the galaxy UGC 6093 was developed sequentially in time based on the parameter $\rho$ equations (20), (21) and (22). Therefore, the spiral patterns at different stages of evolution have different morphologies. It is possible to describe the entire evolution of the spiral pattern with the ROTASE model with a certain time range, while there is no such equivalent method from other current available models. The principle behind this model is equivalent to the motion of an object in Newtonian physics: the past and future motion of the object can be calculated based on its current motion by Newton's laws of motion.

Figure 22 shows the possible spiral pattern evolution of the UGC 6093 from the near past to the near future calculated by the formulas with assumption that the structure and the rotation of the galaxy is stable during such time period. Figure $22 \mathrm{~A}$ is the possible past spiral pattern 
of UGC 6093 at $470^{\circ}$ galactic bar rotation time from current time (roughly about 135 million years ago if using the rotation of the galactic bar of the Milky Way as a reference). At that time in the past, the blue and red line sections had not started yet, and the spiral arms were produced with parameter equation:

$$
\rho=0.624 * \exp (0.002 * \theta)
$$

The total rotation angle of the galactic bar is $240^{\circ}$ with assumption that the maximum radius of the visible spiral arms is the same as the current radius $\mathrm{R}$ shown in Figure 21.

Figure $23 \mathrm{~B}$ is the possible past spiral pattern of UGC 6093 at $270^{\circ}$ galactic bar rotation time from current time (roughly about 80 million years ago), at that time in the past, the blue line section had not started yet.

Red section with $\theta\left(0^{\circ}\right.$ to $\left.200^{\circ}\right)$ :

$$
\rho=0.384+0.0012 * \theta
$$

Yellow section with $\theta\left(201^{\circ}\right.$ to $\left.350^{\circ}\right)$ :
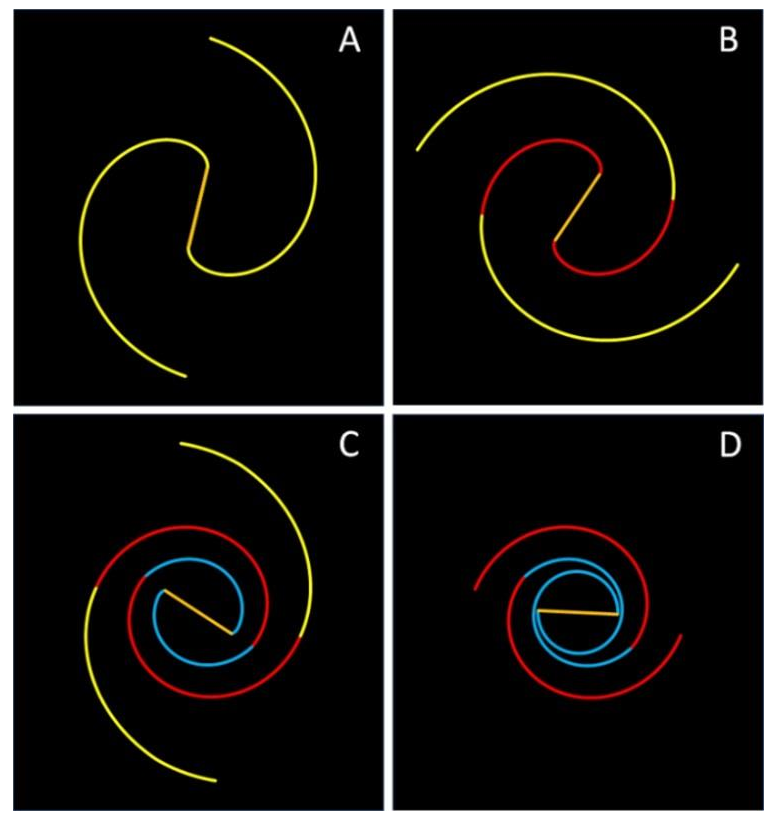

Figure 22: The possible evolution of the spiral arm pattern of UGC 6093. A: 470॰ of bar rotation angle (time) past; B: $270^{\circ}$ of galactic bar rotation angle past; C: Current; D: $150^{\circ}$ of galactic bar rotation angle (time) future.

Figure 22 shows that the spiral pattern of the galaxy UGC 6093 may have been changing continuously during the entire history of its evolution. If the length and curvature of the spiral arms change with the evolution of the galaxy, then its pitch angle should follow such a change also. Figure 23 shows the pitch angle change with the evolution calculated from the Figure 22. One can see that its pitch angle decreases significantly along the evolution timeline. The average pitch angle of a galaxy also depends on the quality of the image. For example, for NGC 4548 shown in Figure 9, the pitch angles from those two different images will be significantly different. It was clearly demonstrated that the pitch angle of a galaxy heavily depends on the length of the spiral arm [18]. The quality of an image heavily depends on how the image is taken such as the

$$
\rho=0.624 * \exp (0.002 *(\theta-200))
$$

Figure 22C is the current spiral pattern of the UGC 6093. Figure 22D is the possible future spiral pattern of UGC 6093 at $150^{\circ}$ galactic bar rotation time from current time with 1.5 loop windings (roughly about 45 million years future).

Blue section with $\theta\left(0^{\circ}\right.$ to $\left.420^{\circ}\right)$ :

$\rho=0.85 * \exp \left(-0.000015 *(\theta-650)^{2}\right)$

Red section with $\theta\left(421^{\circ}\right.$ to $\left.620^{\circ}\right)$ :

$$
\rho=0.384+0.0012 *(\theta-420)
$$

More or less in $150^{\circ}$ of the galactic bar rotation angle time range in the future, only blue and red sections will be visible, the yellow section arms will fade away and disappear due to aging. This projection is based on the fact that current visible spiral galaxies have less than two loop windings, and most long arm galaxies have 1 to 1.5 loop windings. The UGC 6093 will be more tightly wound with smaller visible radius of the spiral arms. wavelength, the photographic exposure time, etc. Such significant dependencies cast a doubt on the possible relationship between the calculated pitch angle of the galaxy and the mass of SMBH. The SALWA pitch angle of the one arm galaxy NGC 4618 is $10.62^{\circ}$, does the pitch angle represent the entire galaxy? It is expected that the average pitch angle of galaxies in Hoag's object family (such as the galaxy ISI961159, galaxies in Figure 8) should be zero or almost zero. The galaxy CGCG 119-82 studied in section 8.1 above has a very tight ring pattern with a strong galactic bar, and the average pitch angle of this type of galaxies with the tight ring pattern is expected to be almost zero, however, such zero pitch angles do not reflect central "super-super" massive black holes. 


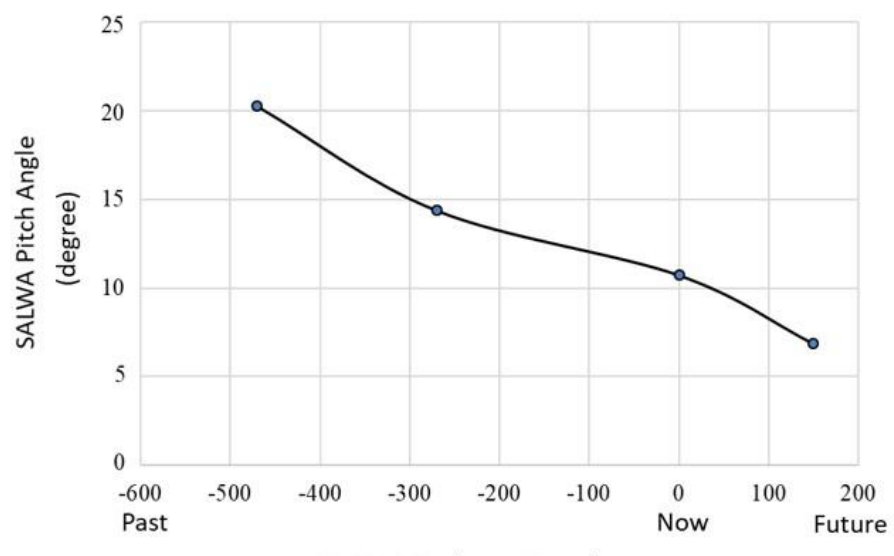

UGC 6093 Evolution Time from Now

(Rotation angle of Galactic Bar-degree)

Figure 23: The pitch angle changes with evolution time of UGC 6093

Readers may think that the description of the possible evolution of the UGC 6093 in Figure 22 just parameterizes the spiral curve, splits the curve into different sections and treats each section separately. However, this is a misunderstanding because the mathematical parameterization of a curve and the simulation of the curve by a physical model have fundamental difference although the two methods use the same mathematical procedure. Theoretically and realistically speaking, one can fit any spiral curve with multi-section method by different mathematical equations without a physical model, but such parametrization is just pure mathematics and does not have any physical meaning. When developing parameterized equations from a physical model, all the parameters and variables in the equations have their unique physical meanings and will describe the characteristics of the curve for why and how. For example, the trajectory of a bullet shot from a gun can be easily fit by many mathematical equations with multiple sections, but such fitting is just a pure mathematics and has no any physical meaning. Only the fitting (or parametrization) by the Newton's law of motion has real physical meaning such as the speed, direction, gravity, wind speed, air friction, distance, etc.

As an illustration, the new spiral formulas derived from this ROTASE model can be used to simulate the spiral patterns of Earth natural spiral objects as shown in Figure 24.
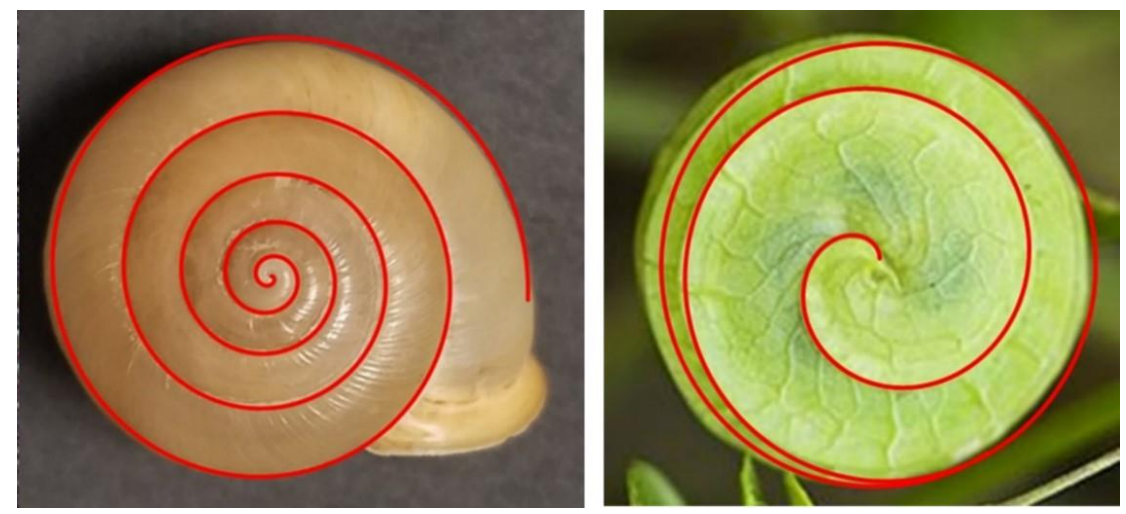

Figure 24: Simulation of spirals of land snail shell (left) and the Medicago Orbicularis seed pod (right) by ROTASE model.

The spiral pattern of the land snail shell can be nicely simulated with the following parameter equation:

$$
\rho=2 *(1+0.0011 * \theta)
$$

And the spiral pattern of the Medicago Orbicularis seed pod can be nicely simulated with the following parameter equation:

$$
\rho=4 * \exp (-0.001 * \theta)
$$

However, the simulation in Figure 24 is just pure mathematics without any physical meanings. The 3 -section simulation of the UGC 6093 in Figure 20 clearly indicates the time sequence that the yellow section was developed first, then, the red section, and the blue section is last (current). The time sequence is the evolution sequence, so we can roughly know what the spiral pattern looks like when only the yellow section was developed, what the spiral pattern looks like after the red section and yellow section were developed and so on. However, this type of description of the possible evolution of the spiral pattern is limited to the available time sequence associated to each section for any galaxies. We cannot deduce the possible spiral pattern in the past before the yellow section and future pattern too far from the current pattern.

\subsection{The possible spiral pattern evolution of the Galaxy M51}

As shown by the original image of M51 and the simulation in section 6, the M51 has two very distinguishable spiral development periods by the yellow 
and red spiral lines. In time sequence, the yellow spiral lines were developed first, the red spiral lines were developed later. The past spiral pattern of M51 at $485^{\circ}$ of galactic bar rotation time in the past is shown in Figure 25 in which only the yellow arms were developed, and the red spiral arms had not started yet. The parameter $\rho$ equation for this period of time is:

$$
\rho=0.00088 *(\theta-50)^{2}+11.704
$$

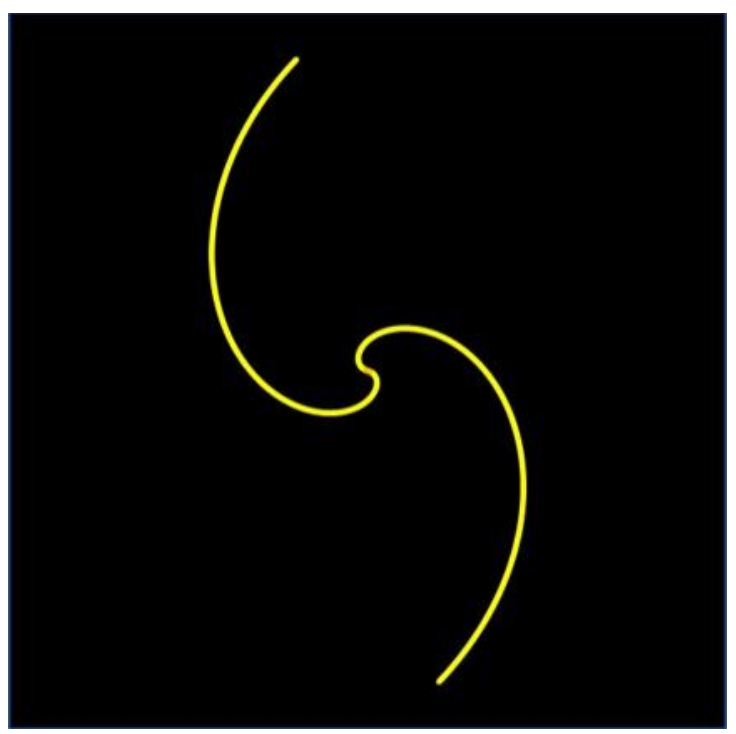

Figure 25: the possible spiral pattern of the M51 at $485^{\circ}$ of galactic bar rotation time in the past from now.

We cannot project the spiral pattern of M51 before the yellow line without some reliable clues (or references). Furthermore, if the M51 and its companion galaxy NGC5195 continue to move closer in the future, then, it will be expected that the yellow line spiral arm will gradually fate away due to aging, but the central symmetry of M51 will be further damaged by the increase of highly unsymmetrical gravitational interaction between the two galaxies.

\section{Milky Way}

The Milky Way is the galaxy in which we reside. There is no way we can get a real image of the whole Milky Way, and the most recent available image is an artist's illustration as shown in Figure 26. The illustration is based on clues from many observations and comparison of such observations with other galaxies. Whether the real morphology of the Milky Way matches the illustration is a question. Based on the illustration, the Milky Way is a barred spiral galaxy with two major leading spiral arms and two minor branches. The two major spiral arms can be nicely simulated shown in Figure 26 by the ROTASE model with the following parameter equation with $365^{\circ}$ of rotation angle:

$$
\rho=0.18 *(1+0.0085 * \theta)
$$

The SALWA pitch angle of the Milky Way is about $12.33^{\circ}$. However, the reliability of this value will depend on the reliability of the artist's depiction of the Milky Way. This pitch angle is about half of the value obtained by Levine et. al. [38], but agrees very well with most of data listed in Vallee's paper [39]. If the illustration roughly reflects the main characteristics of the true Milky Way, then, the parameter $\rho$ equation (31) indicates that the Xmatter emission in the Milky Way is relatively weak and steadily decreases. The spiral arms will be more tightly wound in the future if the current trend continues, such information can only be revealed by the ROTASE model at the moment. While this may indicate that the visible spiral arm size of Milky Way disc could be smaller in the near future, but this does not mean that the real disc size will be smaller. 


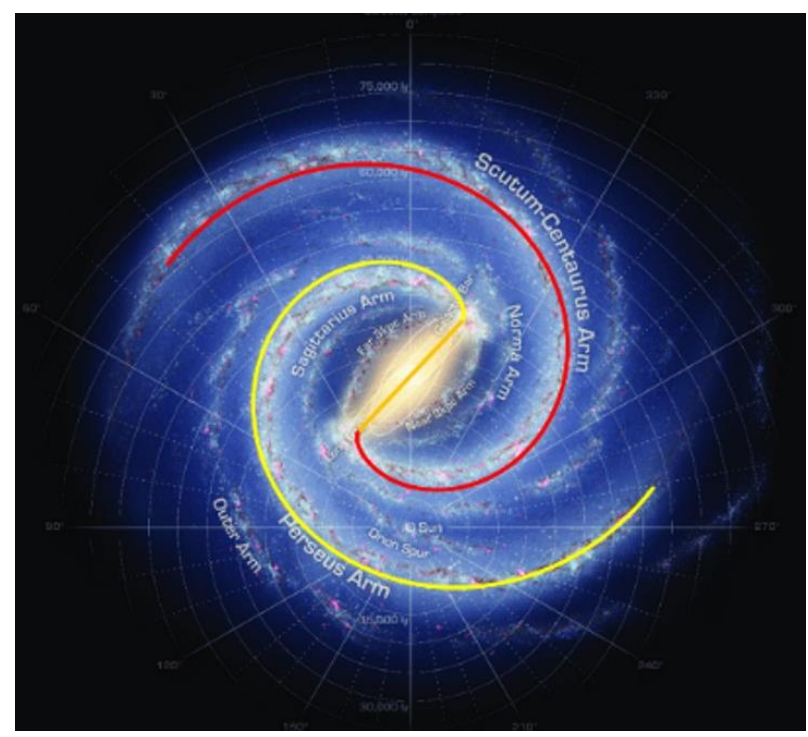

Figure 26: Milky Way and simulation by the ROTASE model, Euler $(43,0,0) . \rho=0.18^{*}\left(1+0.0085^{*} \theta\right)$

\section{Comparison with other spiral equations}

The Archimedean spiral, hyperbolic spiral, Logarithmic spiral and Ringermacher-Mead spiral are commonly used to fit spiral patterns of galaxies [40-44]. The Archimedean spiral produces spirals with equal space between the spiral windings; the hyperbolic spiral has very flat/straight line when the rotation angle is small, the curvature of the spiral gradually increases with the increase of the rotation angle; the logarithmic and Ringermacher-Mead spirals have similar characteristics which produce nice spirals when the rotation angle is small, and the spirals will gradually become flat and approach an asymptotic straight line when the rotation angle increases. The Archimedean, hyperbolic and the logarithmic spirals are limited to a very small portion of spiral galaxies; the Ringermacher-Mead spiral has much broader application with two adjustable parameters. However, all these spiral formulas cannot produce the ring patterns like Figure 1a, Figure 1b, Figure 1f and Figure 1g, and cannot quantify the radius of the ring equivalent to equation (4). In addition, fitting spiral pattern with all those formulas is just pure mathematics without any physical meaning.

\section{Discussion}

The ROTASE model successfully describes the spiral galaxies in many aspects as demonstrated above, specially, the ROTASE model clearly explains the following special patterns:

1. The one side trail effect of the galaxy J101652.52004630.0.

2. The galaxy MCG+00-04-051 with one spiral arm completely broken connection from the galactic bar end, but the other spiral arm is still connected to the end of galactic bar by a weak section of spiral arm.

3. Chain-link arm crossing style in double ring galaxies.

4. The ring crossing points of the galaxy SDSS J015701.50-001644.4 are not located at any Lagrangian points.
5. The galaxy J102942.99-022704.0 with 8-shaped unequal double rings and the ring crossing points are not located at any Lagrangian points.

6. The formation of the one arm galaxy.

7. The formation of the Hoag's object.

The density wave theory, the manifold theory and other available theories cannot explain those special phenomena. Therefore, those models are theoretically possible, but practically implausible at current stage, should be improved if possible. The conversion process of X-matter to hydrogens could be "similar" to the matter conversion process in the Big Bang theory, where the primordial matter released during the Big Bang was converted to the hydrogens. The "similar" here does not means in any aspect that the conversion of the X-matter into hydrogens is the smaller scale of the matter conversion in the Big Bang. The physical conditions (such as pressure, temperature, density, etc..) in the two conversion processes are completely incompatible. This model is in a primary stage and may have many defects and is far from completed. Many new questions may arise. At current stage of physical theories, the black hole has only three externally observable classical parameters (no-hair theorem): mass, electric charge, and angular momentum. Nothing within its horizon can escape from the black hole. The assumption of the X-matter emission by the SMBH directly conflicts with these black hole theories. The proposal of the "X-matter" is very similar to the proposals of "dark matter" and "dark energy". The dark matter was proposed to explain the flat rotation of the galactic disks, and the dark energy with repulsive force was proposed to explain the accelerated expansion of the universe; the current proposed dark matter and dark energy are claimed to be about $95 \%$ of the total matters of the universe, the matter we can observe is only about $5 \%$ of the total matter in the universe. Tremendous amount of resources and efforts have been put into searching such unverified matters/particles in several decades, so far, no success. Critical questions will naturally arise as to how the matter conversion happens inside of the SMBH, and how the X-matter is emitted by the SMBH and later converted to the hydrogens after the emission. There are no answers to those questions currently. Future research by astronomers, cosmologists, high energy physicists and 
particle physicists may provide answers or never have answers. The Big Bang theory was proposed 100 years ago. It was highly speculative at that time without explanation for why and how it happened: if the primordial matter released from the Big Bang in the very beginning had mass or was massless; if that matter was gravitational, nongravitational, or antigravitational; and how the primordial matter was converted to hydrogens and other matters which are the building materials for our universe today. However, after several decades of debate and research with dramatic advancement in high energy physics and particle physics, it has become the leading hypothesis for the universe. Scientists came up with hypotheses to figure out the possible mechanisms for how the initial primordial matter released from the Big Bang was converted to leptons and quarks, and further converted to atoms and started the formation and evolution of stars and galaxies, etc. Understanding the conversion mechanism of X-matter to hydrogens may be more difficult and take more time. Hajdukovic proposed a very interesting hypothesis in which the extremely strong gravitational field deep inside the horizon of the SMBH can create particle-antiparticle pairs from a physical vacuum. The matter and antimatter are repulsive deep inside of the $\mathrm{SMBH}$, and the $\mathrm{SMBH}$ violently repels the antiparticles with antigravity force. The universe is in cycling alternatively by matter and antimatter [45]. The matter cycling by the ROTASE model and the hypothesis by Hajdukovic share the same philosophy in different aspects. The ROTASE model seems consistent with Stephen Hawking's definition. The success of the nice simulation and explanation of various spiral patterns by ROTASE model indicates that everything happens with reasons in the universe; the universe is operated by laws and the mathematics is the execution tool of the laws; the author believes that the spiral patterns of galaxies should be developed by one mechanism not by many mechanisms for the objects with such cosmological scale. This is philosophically consistent with Einstein's principle of simplicity. It is no doubt that the successful description of most (if not all) spiral galaxies by the new set of spiral equations is achieved not by a luck, rather by a mysterious connection between the new set of spiral equations and the real mechanism even if the conjecture of $\mathrm{X}$-matter emission by the SMBH is not compatible with current physics at the moment. We may think "is the current mainstream knowledge absolutely correct? are we restrained?" In the history of science, it has never happened that right mathematical formulas can be derived from an unreasonable/wrong model, such formulas can nicely describe broad observations and make reasonable predictions; such contradictive case will not happen in the future. What we see may not be what the truth is. The most famous example in the science history is the Copernicus' heliocentric theory proposed in $16^{\text {th }}$ century when the geocentric theory was the only theory "correctly" described observation that the Sun, the Moon and all stars in the sky circulated around the Earth, the Earth was the center of universe. The truth is that the Earth and the Moon circulate around the Sun; the Earth and the Sun are the negligible points in the universe, and circulate around Milky Way center, the Milky Way also circulates another object. Another famous example is the Dirac's anti-particle hypothesis, he was laughed for such complete imagination at that time. History shows in many times that today's nonsense could be tomorrow's common sense.
We feel the sweet smell in the dark, and we need to find out where and what it is. The ROTASE model is based on the conjecture that the unknown matter is emitted by the $\mathrm{SMBH}$ located at the galactic center, however, the proposed and popularly accepted SMBH located at the galactic center has been challenged recently by the new study about the geodesic motion of S2 and G2 around the Milky Way galactic center [46], in this interesting study, BecerraVergara et. al., proposed that a quantum core of the fermionic dark matter could be located at the galactic center as alternative to the SMBH. Any new findings are good news to make us better understanding the mysterious heart of the galaxy, the objects in the center of galaxies could be much more complicate and interesting than what we currently know about black holes. The X-matter emission is not necessary from the $\mathrm{SMBH}$, it is just emitted by a mysterious special object at the center of the galaxy, such special object can be called as "black hole" for now or other names in the future, we have to rethink what is really located at the center of the galaxy. Extraordinary ideas need extraordinary approaches. Science is the exploration of educated conjectures, there is no mystery in the universe, only our ignorance. It will be appropriate to finish this paper with Einstein's word: If we knew what it was we were doing, it would not be called research, would it?

\section{Summary}

The proposed ROTASE model has made the following achievements so far:

1. New spiral formulas have been derived which can simulate (or fit) most (if not all) of spiral patterns of disc galaxies. The spiral formulas have one parameter and one variable. They should be the simplest formulas to describe the spiral arm patterns of disc galaxies for now and for the future.

2. It explained the observation that star formation happens simultaneously in the entire spiral arm, and new stars in those areas have a narrow range of the ages.

3. It explained the sharp front edge and rear trail blurry pattern of the spiral arms

4. It explained the special pattern with the broken connection of the spiral arms from the ends of the galactic bar, which is due to the termination of $\mathrm{X}$ mater emission.

5. It predicts that the spiral pattern of the galaxy MCG+00-04-051 in the near future will morphologically look as a "normal" spiral galaxy in which the inner ends of spiral arms will "connect" to the ends of galactic bar instead of being further separated, and the galactic bar will overpass the ends of spiral arms which will make the galactic bar look ahead of the spiral arms.

6. It explained the sequential decrease of the luminosity of the spiral arms along the spiral arm lines from the ends of galactic bars to the ends of visible arms. This is due to the gradual decrease of the amount of the X-matter along the spiral arm line. The distance of the arms to the galactic center, the density and the age of stars are not the main factors for such sequential decrease.

7. It explained the observation of multiple arm crossings of ring galaxies, and demonstrated that 
most (if not all) rings patterns (single ring and double ring) can be described with the universal Gaussian parameter equation. The single spiralring pattern is a special case of the double ring pattern in which the two rings are perfectly merged (overlapped) together.

8. An equation was derived from the model to calculate the radius of the rings for the galaxies with single spiral ring patterns.

9. A formula to calculate the pitch angle of spiral arm is derived from this model. The spiral arm length weighted average pitch angle of the galaxy is proposed for more fairly calculating the average pitch angle for the entire galaxy.

10. It is the first to explain that the double ring patterns like NGC 7098 are actually made of two identical rings and each ring is made of a half inner ring and a half outer ring, the two identical rings are linked with chain-link style arm crossing. This chain-link style arm crossing is caused by the significant difference between the amounts of two X-matter bands which are emitted at significantly different times.

11. It explained the galaxy J102942.99-022704.0 with unequal 8-shaled double rings due to the unequal $\mathrm{X}$-matter emissions at two sides of the SMBH.

12. It is the first to explain that the double ring patterns with 8-shaped inner rings wrapped by larger outer rings like NGC 1079 are actually made of two identical rings, each ring is made of a half large outer ring and multi-section inner rings.

\section{References}

[1] Conselice C.; Wilkinson A.; Duncan K..; Mortlock, A. The evolution of galaxy number density at $\mathrm{z}<8$ and its implications, ApJ, 2016, 830, 83-99.

[2] Hubble, E. The Classification of Spiral Nebulae, The Observatory, 1927, 50, 276-281.

[3] Elmegreen, D.; \& Elmegreen, B. Flocculent and grand design spiral structure in field, binary and group galaxies, MNRAS, 1982, 201,1021-1034.

[4] Elmegreen, D.; Elmegreen, B. Arm Classifications for spiral galaxies. ApJ, 1987, 314, 3-9.

[5] McVitte, G.; Payne-Gaoschkin, C. A model of A spiral galaxy, MNRAS, 1951, 111 (5), 506-522.

[6] Lin, C.; Shu F. On the spiral structure of disk galaxies. ApJ. 1964, $140,646-655$

[7] Shu, F. Six Decades of Spiral Density Wave Theory, Annu. Rev. Astron. Astrophys. 2016, 54, 667-724.

[8] Julian, W.; Toomre, A. Non-axisymmetric responses of differentially rotating disks of stars, ApJ. 1966, 146, 810-830.

[9] Romero-Gómez, M.; Masdemont, J.; Athanassoula, E.; GarcíaGómez, C. The origin of rR1 ring structures in barred galaxies, A\&A 2006, 453, 39-45.

[10] Romero-Gómez, M.; Athanassoula, E.; Masdemont, J.; GarcíaGómez, C. The formation of spiral arms and rings in barred galaxies, A\&A, 2007, 472, 63-75.

[11] Athanassoula, E.; Romero-Gómez, M.; Masdemont, J.; Rings and spirals in barred galaxies - I. Building blocks, MNRAS, 2009, 394(1), 67-81.

[12] Athanassoula, M.; Romero-Gómez, M.; Bosma, A.; Masdemont, J. Rings and spirals in barred galaxies - II. Ring and spiral morphology, MNRAS, 2009, 400(4), 1706-1720.

[13] Athanassoula, E.; Romero-Gómez, M.; Bosma, A.; Masdemont, J. Rings and spirals in barred galaxies - III. Further comparisons and links to observations, MNRAS, 2010, 407(3), 1433-1448.

[14] Dobbs, C.; Theis, C.; Pringle, J.; Bate, M. Simulations of the grand design galaxy M51: a case study for analysing tidally induced spiral structure Mon. Not. R. Astron. Soc. 2010, 403, 625-645.
13. It explained that the formation of the Hoag's object is due to the termination of the X-matter emission of single ring galaxies, and its possible pattern evolution sequence is illustrated.

14. It explained the formation of one-arm galaxies in which one side of the SMBH has weak or no Xmatter emission, but other side has strong $\mathrm{X}$ matter emission.

15. It provides a method to deduce the possible evolution of the spiral patterns of galaxies where spiral arms have distinguishable sections associated with different time periods.

16. The results from the ROTASE model indicate that the average pitch angle of the spiral galaxies heavily depends on the length of the spiral arms and the quality of the images of galaxies. The average pitch angle also changes with the evolution of the galaxies; such dependence will make the attempt to correlate the average pitch angle with the mass of the central SMBH more difficult and unreliable.

17. It indicates that the matter in the universe may be recyclable in some extent.

18. The results from the ROTASE model indicate that the winding of the spiral arms of the Milky Way may become tighter in the future if the current artist's illustration is reliable.

[15] Dobbs, C,; Baba, J. Dawes Review 4: Spiral Structures in Disc Galaxies, PASA, 2014, Vol. 31, e035.

[16] Pan, H. New formulas and mechanism for the spiral arm formation of Galaxies. IJP., 2019. 7, 73-85.

[17] Pan, H. Application of new formulas for the spiral arm formation to selected galaxies with special patterns. A JAA. 2020, 8, 45-66.

[18] Pan, H. Pitch angle calculation of spiral galaxies based on the ROTASE model. IPJ. 2021, 9, 71-82.

[19] Hawking, S. A brief history of time, (Bantam Books, NY, NY), 1998, pp 10. ISBN 0-553-10953-7.

[20] Grand, R. J. J., Kawata D., Cropper M., Spiral arm pitch angle and galactic shear rate in N-body simulations of disc galaxies, A\&A, 2013, 553, A77.

[21] Berrier, J. C., Davis B. L., Kennefick D. et al., Further Evidence for a Supermassive Black Hole Mass-Pitch Angle Relation, ApJ, 2013, $769,132$.

[22] Ringermacher, H. and Mead, L., Simple fit of data relating supermassive black hole mass to galaxy pitch angle, AJ, 2009, 137, 4716-4717.

[23] Seigar, M.; James, P. The structure of spiral galaxies-II. Nearinfrared properties of spiral arms", MNRAS. 1998, 299, 685-698.

[24] Walmsley, M. et al.; Galaxy Zoo DECaLS: Detailed Visual Morphology Measurements from Volunteers and Deep Learning for 314,000 Galaxies, 2021, https://arxiv.org/abs/2102.08414v1

[25] Contopoulos, G.; Grosbol, P. Stellar dynamics of spiral galaxies: nonlinear effects at the $1 / 4$ resonance, Astron. Astrophys. 1986, 155, 11-23.

[26] Buta, R. Resonance Rings and Galaxy morphology, Astrophys. Space Sci. 1999, 269-270: 79-99.

[27] Buta, R., The catalog of southern ringed galaxies, ApJS. 1995, 96, 39-116.

[28] Buta, R., Galactic rings revisited. II. Dark gaps and the locations of resonances in early-to-intermediate-type disc galaxies, MNRAS. 2017, 470, 3819-3849.

[29] Buta, R.; Byrd, G. A Hubble space telescope study of star formation in the inner resonance ring of NGC 3081. AJ. 2004, 127, 19822001.

[30] Buta, R.; Burcell, G. NGC 3081: Surface photometry and kinematics of a classic resonance ring barred galaxy. AJ, 1998, 115, 484-501. 
[31] Hoag, A. A., A peculiar object in Serpens, Astron. J., 1950, 55, 170.

[32] Brosch, N., The nature of Hoag's object: the perfect ringed galaxy, Astron. Astrophys. 1985, 153, 199-206.

[33] Toomre, A., in Evolution of galaxies and stellar populations, ed. R. B. Larson and B. M. Tinsley (New Havenn: Yale University Observatory), $\mathrm{p} 401$.

[34] Theys, J.C., and Spiegel, E. A., Ring Galaxies II. ApJ., 1977, 212, 616-633.

[35] Schweizer, et. al., The structure and evolution of Hoag's object, AJ., 1987, 320 454-463.

[36] Kaczmarek, J. and Eric M. Wilcots, E., High-resolution H i distributions and multi-wavelength analysis of magellanic spi-rals NGC 4618 AND NGC 4625, The Astronomical Journal, 2012, 144:67 (13pp).

[37] Sellwood J.; Carlberg R. Spiral instabilities: mechanism for recurrence, MNRAS. 2019, 489, 116-131.

[38] Levine, E., Blitz, L., Heiles, C., The Spiral Structure of the outer Milky Way in Hydrogen, Science, 2006, 312,1773-1777.

[39] Jacques P. Vallée, The Spiral Arms and Interarm Separation of the Milky Way: An Updated Statistical Study, AJ. 2005, 130, 569-575.
[40] Ma J., A Method of Obtaining the Pitch Angle of Spiral Arms and the Inclination of Galactic Discs, Chin. J. Astron. Astrophys., 2001, $1,395$.

[41] Seigar M. S., Kennefick D., Kennefick J., Lacy C. H. S., Discovery of a relationship between spiral arm morphology and supermassive black hole in disk galaxies, ApJ, 2008, 678, L93.

[42] Karachentsev I. D., Karachentseva V. E., Commun. Byurak. Obs., $1967,38,47$.

[43] Kennicutt, R. C., Jr. The shapes of spiral arms along the Hubble sequence, AJ. 1981, 86, 1847-1858.

[44] Ringermacher H., Mead L., 2009, A new formula describing the scaffold structure of spiral galaxies, MNRAS, 2009, 397, 164-171.

[45] Hajdukovic, D.S. Do we live in the universe successively dominated by matter and antimatter? Astrophys Space Sci. 2011, 334, 219-223.

[46] Becerra-Vergara, E., Argüelles, C., Krut1, A., Rueda, J., and Ruffini, R. Geodesic motion of S2 and G2 as a test of the fermionic dark matter nature of our Galactic core, A\&A 2020, 641, A34. 